\title{
SEGUIMIENTO DE AVES ACUÁTICAS AMENAZADAS EN LAGUNAS DE LA RESERVA DE LA BIOSFERA DE LA MANCHA HÚMEDA (ESPAÑA CENTRAL)
}

\author{
Rafael GOSÁLVEZ ${ }^{(a)}$ - José A. GIL-DELGADO ${ }^{(b)}$ - Carmen VIVES- \\ FERRÁNDIZ ${ }^{(\mathrm{b})}$ - Gema SÁNCHEZ ${ }^{(\mathrm{a})}$ - Máximo FLORÍN ${ }^{(\mathrm{c})}$ \\ (a) IHE-Grupo de Investigación en Hidroecología. Departamento de Geogra- \\ fía y Ordenación del Territorio, Universidad de Castilla-La Mancha \\ (b) Unidad de Investigación de Vertebrados Terrestres, Institut Cavanilles de \\ Biodiversitat i Biologia Evolutiva, Universitat de València. \\ (c) IHE-Grupo de Investigación en Hidroecología. Centro Regional de Estu- \\ dios del Agua (CREA), Universidad de Castilla-La Mancha (UCLM).
}

Recibido: 16/04/2011

Devuelto: 06/06/2011

Aceptado: 22/06/2011

RESUMEN: La Reserva de la Biosfera de La Mancha Húmeda es el principal grupo o red de lagunas de interior de la Península Ibérica donde poblaciones de aves acuáticas, con prioridad de conservación a nivel europeo, nidifican e invernan constituyendo un punto caliente para la conservación de la biodiversidad. En la actualidad, es uno de los complejos de humedales más amenazados en España y Europa debido a las actividades humanas, principalmente la agricultura, hasta el punto que en el año 2008 se propuso su descatalogación como Reserva de la Biosfera. Entre enero de 2007 y diciembre de 2010 se ha realizado un censo mensual en 10 lagunas localizadas en el Campo de San Juan (SE de Toledo, SO de Cuenca y NE de Ciudad Real) dentro de la Reserva de la Biosfera, presentando en este trabajo los resultados del seguimiento de las 12 especies catalogadas como SPEC 1 y 2 (BIRDLIFE INTERNATIONAL, 2004) reconocidas en este espacio geográfico. Los resultados muestran que dos especies, el porrón europeo (Aythya ferina) y la cigüeña común (Ciconia ciconia) son comunes y se encuentran ampliamente distribuidas en las lagunas estudiadas, siendo el porrón europeo la especie que ha mostrado el mayor tamaño poblacional. Cuatro especies (Ciconia nigra, Platalea leucorodia, Marmaronetta angustirostris y Numenius arquata) tienen una presencia ocasional y con muy bajos tamaños poblacionales, utilizando estas lagunas como lugar de descanso en sus rutas migratorias o movimientos dispersivos El resto de especies (Vanellus vanellus, Limosa limosa, Tringa totanus y Philomachus pugnax) presentan escasos efectivos demográficos, utilizando las lagunas de manera ocasional y distribuyéndose de manera discontinua. Cinco lagunas (Manjavacas, Camino de Villafranca, La Veguilla, Salicor y Pedro Muñoz) han presentado en algún momento del periodo de seguimiento de 8 a 10 de las especies inventariadas, todas ellas bajo protección legal como Reserva Natural. La profundidad de la lámina de agua, la presencia de orillas adecuadas, el desarrollo de helófitos, la presencia de nutrientes y las ca- 
racterísticas hidroquímicas parecen condicionar el uso de las lagunas por las diferentes especies.

PALABRAS CLAVE: Zoogeografía, Reserva de la Biosfera "La Mancha Húmeda", aves acuáticas amenazadas, seguimiento, conservación.

\title{
MONITORING WATERBIRDS UNDER THREAT IN the LAKES of THE "MANCHA HÚMEDA" BIOSPHERE RESERVE (CENTRAL SPAIN)
}

\begin{abstract}
The Mancha Húmeda Biosphere Reserve is the main group of inland shallow lakes in the Iberian Peninsula, where priority aquatic birds species for conservation in the European range breed and winter. Nowadays, this Reserve is among the most threatened wetland districts in Europe due to human activities, mainly agriculture; as, its status as a Biosphere Reserve is under revision since 2008. Monthly censuses were performed in 10 shallow lakes of the Biosphere Reserve from January 2007 to December 2010; results reported here focuse on the 12 species belonging to SPEC 1 and 2 categories. The results show that two species (Aythya ferina and Ciconia ciconia) are common and are widely distributed in the shallow lake studied; Aythya ferina is the specie that has shown the greatest population size. Four species (Ciconia nigra, Platalea leucorodia, Marmaronetta angustirostris and Numenius arquata) had an occasional presence and a low population size, using the shallow lake as a resting place on their routes or movements dispersed. The remaining species (Vanellus vanellus, Limosa limosa, Tringa totanus and Philomachus pugnax) have few demographic effects, using occasional the shallow lake and discontinuosly distributed. Five shallow lakes (Manjavacas, Camino de Villafranca, La Veguilla, Salicor and Pedro Muñoz) presented sometime in the monitoring period of 8 to 10 species recorded, all of them under legal protection as a Nature Reserve. Water column depth, presence of shores, development of helophytes, presence of nutrients and hydrochemical characteristics seem to control the use of these wetlands by the different species.
\end{abstract}

KEY WORDS: Zoogeography, Biosphere Reserve "La Mancha Húmeda", threatened aquatic birds, monitoring, conservatio.

\section{INTRODUCCIÓN}

La gestión de espacios naturales y de especies constituye la base de las políticas de conservación de la biodiversidad a través de la declaración de áreas protegidas y de la elaboración de listas de especies amenazadas. Una de las formas más usuales de medir la biodiversidad en un determinado lugar es el número de especies, pues está correlacionada con la diversidad ecológica y funcional (GASTON, 1996; NOGUÉS-BRAVO, 2003). En el caso de las listas de especies, probablemente la Lista o el Libro Rojo de la UICN (Unión Internacional para la Conservación de la Naturaleza) es la más conocida a nivel global, constituyendo una de las herramientas principales para la conservación de la fauna y flora, al evaluar de manera objetiva la probabilidad de extinción de una especie en un tiempo dado (MADROÑO et AL., 2005).

En 1994 se desarrolla una lista específica para evaluar el estado de conservación de las aves de Europa atendiendo a los criterios contenidos en el artículo 4.1. de la Directiva Aves, lista que es elaborada por Birdlife Interna- 
cional bajo el título de Birds in Europe (TUCKER y HEATH, 1994). Desde su publicación, esta lista ha sido utilizada ampliamente para determinar prioridades de conservación de este grupo de vertebrados en toda Europa (Birdlife Internacional, 2004). Hay que señalar que esta lista abordó el tema desde el punto de vista del estado de conservación, que no es sinónimo de probabilidad de que la especie se extinga, tal y como lo concibe la UICN. En este sentido, numerosas especies de aves ampliamente distribuidas en Europa, sin correr riesgo de extinción a medio y largo plazo, han sufrido declives significativos en el tiempo debido a la degradación o a la pérdida de hábitats (BIRDLIFE INTERNACIONAL, 2004).

La revisión de Birds in Europe del año 2004 procuró reconciliar el concepto de probabilidad del riesgo de extinción y el de estado de conservación, de manera que ahora para evaluar el estado de conservación se tienen en cuenta también los criterios de la Lista Roja de la UICN (UICN, 2001). Además, Birds in Europe ha desarrollado criterios cuantitativos propios para identificar las Especies que requieren medidas de conservación en Europa (SPEC en sus siglas en inglés). Cada especie es asignada a una de las cinco categorías establecidas: SPEC 1, 2, 3 No SPEC $^{\mathrm{E}}$ y NO SPEC. De las 524 especies evaluadas, $226(43 \%)$ presentan un Estado de Conservación Desfavorable en Europa, clasificándose $40(7,6 \%)$ de ellas como SPEC 1, aquellas especies con mayor necesidad de adoptar medidas de conservación, 45 (8,6\%) como SPEC 2 y $141(26,9 \%)$ como SPEC 3.

Sea cual sea el tipo de lista que se maneje para evaluar el estado de conservación de las especies, siempre se basa en el empleo de criterios cualitativos y/o cuantitativos, entre los que se suelen incluir la tendencia de sus poblaciones, el área de distribución que ocupa, el tamaño poblacional y el número de subpoblaciones que la componen, por citar algunos.

La zoogeografía, por su carácter transdisciplinar entre la biología y la geografía, puede contribuir a establecer esos criterios sobre el estado de conservación de las especies y a elaborar los programas de seguimiento necesarios para poder poner en práctica los mismos. La zoogeografía, al igual que los datos de seguimiento en el tiempo necesarios para evaluar el estado de conservación de una especie, requiere de plazos largos de trabajo mediante un trabajo secuenciado y repetido, lo que va a permitir captar las variaciones temporales y espaciales de las especies sometidas a seguimiento (RUBIO y BEJARANO, 2001) y con ello evaluar su estado de conservación.

En este trabajo se presentan los resultados del seguimiento a lo largo de cuatro años de las especies amenazadas atendiendo a los criterios de Birds in Europe (BIRDLIFE INTERNACIONAL, 2004), en una muestra de diez lagunas 
que se localizan en el núcleo central de la única Reserva de la Biosfera con la que cuenta la comunidad autónoma de Castilla-La Mancha, la Reserva de la Biosfera de La Mancha Húmeda. Esta Reserva es, hoy por hoy, el principal conjunto de humedales del interior de la Península Ibérica, funcionando como un punto caliente (hotspot) para la conservación de la biodiversidad, al albergar especies prioritarias de aves acuáticas tanto en la época de invernada como en la de reproducción. Paradójicamente, es uno de los complejos de humedales más amenazados por la actividad humana, principalmente la agricultura de regadío, hasta el punto de que en el año 2008 los principales grupos ecologistas de España propusieron su descatalogación como Reserva de la Biosfera. El objetivo final de este artículo es evaluar la importancia de estas lagunas como refugio para especies amenazadas de aves acuáticas siguiendo los criterios de Birds in Europe.

\section{CARACTERIZACIÓN DEL ÁREA DE ESTUDIO}

\section{II.1. Reserva de la Biosfera de La Mancha Húmeda}

La Mancha Húmeda constituye el mayor sistema palustre del interior peninsular al albergar más de 30.000 ha de humedales (DA CRUZ, 1986) caracterizados por una elevada diversidad fisiográfica y ecológica, directamente relacionada con una de las mayores cuencas continentales terciarias de la Península Ibérica, la Llanura Manchega (CASADO y MONTES, 1995; FLORín, 2001). Localizada la mayor parte en la cuenca alta del río Guadiana, se trata de la principal zona húmeda esteparia, atendiendo a valores climáticos, de Europa Occidental, recordando a algunas zonas húmedas del Norte de África y Asia Central (MARGALEF, 1947).

Frente a otros complejos húmedos de la Península Ibérica, La Mancha Húmeda parece ser el único que tendría un genuino carácter regional (PlANS, 1969). Lo que hace marcadamente diferente a la Mancha Húmeda es su gran extensión superficial, densidad y diversidad. Características específicas adicionales son la naturaleza continental de sus sedimentos, la dominancia de calizas y margas en la litología y el relieve extremadamente llano del área. La baja densidad de drenaje y el clima semiárido Mediterráneo que caracteriza la planicie manchega han llevado a creer que el origen de este complejo humedal fue endorreico (DANTín, 1932 y 1940).

Siete sectores pueden ser distinguidos dentro de La Mancha Húmeda (FIGURA 1): lagunas del SE de Toledo, Mota del Cuervo, Sierra de Altomira, Las Tablas de Daimiel y humedales de su entorno, Campo de Montiel (incluyendo las lagunas de Ruidera), las lagunas volcánicas del Campo de Calatrava y las lagunas de La Mancha oriental. La interacción con grandes siste- 
mas acuíferos, las características locales de las formas del relieve, las relaciones entre humedales y redes de drenaje y las características geoestructurales y litológicas de la formación de las cuencas vertientes y de las cubetas constituyen las bases para la caracterización de estos siete sectores identificados. Son numerosos los procesos geomorfológicos que se han descrito como responsables de la formación de las cubetas lagunares, destacando entre ellos la deflación eólica, los procesos kársticos de disolución y precipitación, los procesos fluviales, los tectónicos y estructurales, los procesos hidromagmáticos y la interacción con las aguas subterráneas. La contribución de la precipitación, la escorrentía y el flujo de aguas subterráneas para cada humedal es también variable, dando lugar a distintos tipos de alimentación.

El tipo de humedal más común identificado son las llanuras de inundación fluvial y las lagunas. En éste último caso, se trata de humedales esteparios someros y temporales con una pequeña cuenca vertiente. Estas lagunas suelen presentar un cinturón marginal de freatofitas y/o halófitas y una lámina de agua abierta ocupada con diferentes productores primarios (macrófitos sumergidos, fitoplancton y fitobentos). Se han inventariado 167 lagunas que cubrirían unas 5.000 ha, aunque en la actualidad el $60 \%$ se encuentran desaparecidas físicamente o han perdido su funcionalidad hidrológica.

El mayor impacto que ha producido la actividad humana en la Mancha Húmeda es la reducción de la lámina de agua, lo que afecta a la calidad y a la cantidad del agua disponible en cada humedal (FLORín et AL., 1993). Entre las causas principales se encuentran la canalización de las corrientes superficiales, la extracción directa de agua y la sobreexplotación de los acuíferos.

La importancia ambiental de este conjunto de humedales, especialmente por su elevada hidrodiversidad, junto a la presión antrópica negativa ejercida por la agricultura, que ha sobreexplotado los acuíferos y ha eliminado numerosas cubetas hasta borrarlas del mapa, llevaron a la designación en el año 1981 de una parte de las lagunas de La Mancha Húmeda como Reserva de la Biosfera por parte del Programa MAB de la UNESCO a petición del Gobierno Español. Pero la información contenida en la propuesta de declaración presentaba grandes vacíos en temas claves como los propios límites concretos de la Reserva y su zonificación, lo que llevó en enero de 2009 a la Junta de Comunidades de Castilla-La Mancha a lanzar una propuesta de modificación de la Reserva de la Biosfera (JCCM, 2009), que fue contestada por sectores científicos y ecologistas debido a la exclusión de numerosas lagunas como las de origen volcánico del Campo de Calatrava o gran parte de las llanuras de inundación de los ríos Guadiana, Cigüela y Záncara. 
Figura 1. Localización de la Reserva de la Biosfera de "La Mancha Húmeda", sectores reconocidos en ella y lagunas estudiadas.

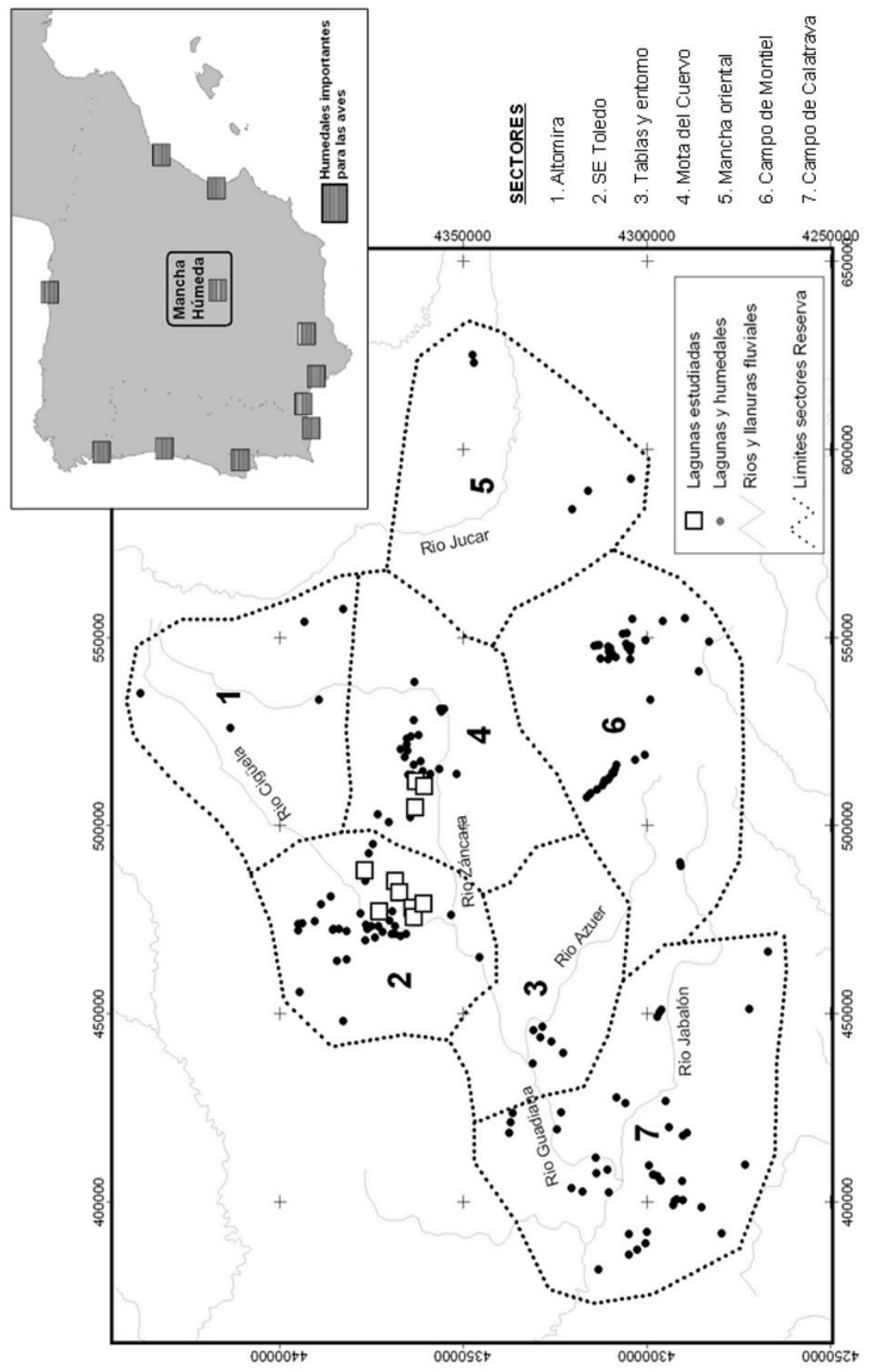

Polígonos. Revista de Geografía, 22 (2012); pp. 89-122 


\section{II.2. Lagunas seleccionadas para el programa de seguimiento}

De todo el conjunto de lagunas, en este trabajo se ha optado por seleccionar una muestra de 10 lagunas localizadas en la parte central de La Mancha Húmeda (sectores de Mota del Cuervo y SE de Toledo), también conocido como lagunas del Campo de San Juan (JIMÉNEZ et AL., 1992). Estas lagunas son las que muestran un hidroperiodo más estable en el tiempo, de estacional a permanente.

Además de incluirse en la Reserva de la Biosfera, todas ellas forman parte de la Red Natura 2000 (LIC y ZEPA "Humedales de La Mancha"), todas se encuentran catalogadas como IBA ( $\mathrm{n}^{\circ} 195$ "Complejo Lagunar de Alcázar de San Juan-Quero" y n ${ }^{\circ} 196$ "Complejo Lagunar de Pedro MuñozManjavacas") y, finalmente, casi todas se encuentran catalogadas como Reserva Natural en el marco de la Red de Áreas Protegidas de Castilla-La Mancha, con la excepción de las lagunas Grande de Quero, Mermejuela y Pajares.

En el CUADRO 1 se muestran las características ambientales de este conjunto de lagunas. La superficie de todas ellas suma 844 ha, con un rango muy variado que va desde las 9 ha de la laguna de Mermejuela a las 230 ha de la laguna de Manjavacas, aunque la mayoría se encuentra entre las 20 y 80 ha de extensión superficial. Algo similar sucede con la profundidad máxima que varía entre los 30 y los $200 \mathrm{~cm}$, aunque la mayor parte de ellas se encuentran por debajo de los $100 \mathrm{~cm}$, tanto de profundidad máxima como habitual. Estas variables morfométricas nos indican que estamos ante lagunas y lagos en función del área y lagunas y humedales en función de la profundidad.

En cuanto a las características hidroquímicas de las lagunas, destacar que no hay ninguna laguna cuyas aguas se califiquen como dulces, presentando todas ellas algún grado de salinidad, desde aguas hiposalinas (cinco lagunas) hasta hipersalinas (lagunas Grande de Quero y Las Yeguas). Los aniones mayoritarios son los sulfatos y los cloruros, mientras que los cationes dominantes son el magnesio y el sodio, por lo que el tipo iónico mayoritario es el sulfatado clorurado-magnésico sódico, es decir, aguas mixtas evolucionadas típicas de lagunas salinas esteparias. En cualquier caso, hay que recordar que la proporción iónica de las aguas puede cambiar a lo largo del año, sobre todo en lagunas fuertemente mineralizadas como las aquí estudiadas (Cirujano y MEDinA, 2002). La concentración y el tipo de sales disueltas en el agua son factores claves que afectan a la flora y a la fauna relacionada con las lagunas. 
Cuadro 1. Caracterización ambiental de las lagunas estudiadas de la Reserva de la Biosfera de "La Mancha Húmeda"

\begin{tabular}{|c|c|c|c|c|c|c|c|c|c|c|c|c|}
\hline \multirow[b]{2}{*}{ Lagunas } & \multicolumn{2}{|c|}{ Morfometría } & \multicolumn{8}{|c|}{ Hidroquímica } & \multicolumn{2}{|c|}{ Ambientes } \\
\hline & $\begin{array}{c}A \\
(h a)\end{array}$ & $\begin{array}{l}\text { P. máx. } \\
\text { (cm) }\end{array}$ & $\begin{array}{l}\text { Cond. } \\
\mu \mathrm{S} / \mathrm{cm}\end{array}$ & $\begin{array}{c}\mathrm{Cl}- \\
\mathrm{meq} / \mathrm{l}\end{array}$ & $\begin{array}{l}\mathrm{SO} 4= \\
\mathrm{meq} / \mathrm{l}\end{array}$ & $\begin{array}{c}\mathrm{CO}_{3} \mathrm{H}- \\
\mathrm{meq} / \mathrm{l}\end{array}$ & $\begin{array}{l}\mathrm{Ca}++ \\
\mathrm{meq} / \mathrm{l}\end{array}$ & $\begin{array}{c}M g++ \\
m e q / l\end{array}$ & $\begin{array}{c}K+ \\
\text { meq/l }\end{array}$ & $\begin{array}{l}\mathrm{Na}+ \\
\mathrm{meq} / \mathrm{l}\end{array}$ & Helófitos & Playas \\
\hline Alcahozo & 70 & 30 & 23.900 & 852,8 & $1.322,2$ & 59,7 & 175,4 & $1.479,5$ & 69 & 521,5 & $\mathrm{~N}$ & $S$ \\
\hline Camino de Villafranca & 140 & 150 & 17.920 & 186,2 & 184,6 & - & 17,5 & - & - & - & $S$ & S \\
\hline Manjavacas & 230 & 60 & 5.740 & 234,9 & 71,6 & 354,2 & 40 & 327,6 & 16,4 & 274,4 & S & S \\
\hline Mermejuela & 9 & 60 & - & - & - & - & - & - & - & - & $\mathrm{N}$ & $\mathrm{N}$ \\
\hline Pajares & 22 & 50 & 30.500 & 189,2 & 321,9 & 0,2 & 56 & 287,6 & 10,2 & 130,5 & $\mathrm{~N}$ & S \\
\hline Pedro Muñoz & 36 & 80 & 6.857 & 46,1 & 55,2 & 3,6 & 32,1 & 32,5 & 21,3 & 20,8 & $S$ & $\mathrm{~N}$ \\
\hline Quero & 78 & 200 & 71.400 & 102,7 & 81,1 & 82,4 & 26,5 & 41,2 & 77,0 & - & $\mathrm{N}$ & S \\
\hline Salicor & 57 & 200 & 9.550 & $1.539,0$ & $5.111,5$ & 37,2 & 651 & $5.141,4$ & 178,5 & 804,4 & $\mathrm{~N}$ & S \\
\hline Veguilla & 136 & 40 & 2.720 & 12,6 & 8,5 & - & 3,1 & - & - & - & S & $\mathrm{N}$ \\
\hline Las Yeguas & 66 & 50 & 71.600 & 246,6 & 323,7 & 8,1 & 70 & 297,8 & 28,2 & 304,3 & $\mathrm{~N}$ & S \\
\hline
\end{tabular}

Por último, se han tenido en cuenta dos variables cualitativas que nos informan de la existencia de diferentes ambientes que determinan la presencia de ciertas especies de aves acuáticas especializadas. Nos estamos refiriendo a la presencia o no de importantes masas de helófitos en los bordes de la lámina de agua o invadiendo la misma, con una cobertura que favorezca a las aves propias de marjales (gallinetas, aguiluchos laguneros, ardeidas, etc.), así como la presencia o no de playas que van a permitir el aprovechamiento de las mismas por aves limícolas. El carácter fuertemente salino de la mayor parte de las lagunas, así como la presión que impone la actividad agraria cultivando hasta el borde de las orillas, impide en general el desarrollo de importantes coberturas de helófitos, que queda restringida a las lagunas que reciben vertidos de aguas residuales depuradas (lagunas de Pedro Muñoz, La Veguilla y Manjavacas), mientras que las playas son relativamente comunes en unas lagunas cuyas cubetas tienden a presentar líneas de costa poco pronunciadas, aunque también se ha detectado la pérdida de estas playas debido a la aparición de taludes generados por la erosión de la lámina de agua por la acción del viento (por ejemplo, la laguna del Camino de Villafranca).

\section{II.3. Situación hidroclimática en los años 2007 a 2010}

La primera consideración a tener en cuenta para comprender la situación hidroclimática de un conjunto de lagunas es caracterizar el clima del territorio en el que se insertan y determinar el comportamiento de las precipitaciones, responsables en gran medida del régimen hidrológico junto a los flujos de agua superficial o subterránea que pueda recibir.

Siguiendo a PEINADO (2000), autora que trabajó con los datos del observatorio meteorológico de Alcázar de San Juan en la serie temporal 1961-1998, la temperatura media anual es de $14,8^{\circ} \mathrm{C}$, con una amplitud térmica media 
de $20,5^{\circ} \mathrm{C}$ y absoluta de $56^{\circ} \mathrm{C}$, lo que nos habla de la existencia de fuertes contrastes estacionales. Las temperaturas más altas se dan en el mes de julio y agosto, produciéndose las más bajas de diciembre a febrero. La precipitación media anual para la serie mencionada ha sido de $387,4 \mathrm{~mm}$, lo que tipifica climáticamente a este observatorio meteorológico como semiárido o incluso árido. Sin embargo, este valor promedio enmascara una gran irregularidad interanual, pues se han registrado desde $569 \mathrm{~mm}$ el año más húmedo (1962) hasta 183,8 mm para el año más seco (1994). Un dato a destacar es la escasez de precipitaciones durante la década de 1990, en donde a excepción de los años 1996 y 1997, no se ha superado en ningún año la media de la serie, obteniéndose además los valores más bajos de la misma (PEINADO, 2000). En cuanto a la distribución de las precipitaciones por estaciones, destaca la primavera como la más lluviosa $(31,2 \%)$, seguida del invierno $(30,4 \%)$ y del otoño $(25,4 \%)$, siendo la estación más seca el verano, donde apenas se supera el 10\% de las precipitaciones (PEINADO, 2000).

Para analizar la situación concreta de los cuatro años de seguimiento que hemos abordado en este trabajo, se han empleado los datos proporcionados por el observatorio meteorológico de Herencia del Servicio Integral de Asesoramiento al Regante de la Consejería de Agricultura y Medio Ambiente del gobierno regional (JCCM, 2011). La precipitación media anual para este periodo de cuatro años solo ha superado el valor promedio hallado por PEINADO (2000) en el año 2010 con 523 mm, si bien el resto de años han presentado valores próximos al promedio ( $386 \mathrm{~mm}$ en 2007 ; 372,4 en 2008 y 341 en 2009). En la figura 2 se muestra la evolución mensual de la precipitación para el observatorio SIAR de Herencia en los años de seguimiento, detectándose una clara tendencia cíclica en la que las precipitaciones más bajas se producen en los meses estivales, como es de esperar en un clima mediterráneo, contribuyendo cada año de una manera distinta el resto de estaciones climáticas (primavera y otoño en el 2007, 2008 y 2009 y el invierno en 2010).

Un suceso meteorológico de gran trascendencia ambiental en el funcionamiento de una parte de las lagunas estudiadas tuvo lugar en la semana del 20 al 26 de mayo de 2007, cuando el Campo de San Juan se vio afectado por una tormenta "supercélula", fenómeno meteorológico poco frecuente que descargó una precipitación máxima de $240 \mathrm{~mm}$ en 24 h (al sur del término municipal de Alcázar de San Juan), unos $130 \mathrm{~mm}$ en el observatorio meteorológico de Alcázar de San Juan y unos $100 \mathrm{~mm}$ en la de Herencia (SÁNCHEZ et $A L ., 2008$ ).

Este suceso produjo una fluctuación extraordinaria de la lámina de agua en las lagunas de Alcázar de San Juan, Quero y Salicor. En el caso de esta últi- 
ma laguna, la profundidad máxima de la columna de agua pasó de $16 \mathrm{~cm}$ en abril de 2007 a más de $250 \mathrm{~cm}$ a principios de junio de 2007, inundándose una superficie de unas 100 ha, el doble de la propia cubeta lagunar (52.25 has) (SÁNCHEZ et $A L ., 2008)$. La conductividad eléctrica del agua pasó de 90 a $2.44 \mathrm{mS} \mathrm{cm}^{-1}$, la turbiedad de 166 a 9.47 NTU, y la salinidad de 172 a 2.38 $\mathrm{mg} \mathrm{L}^{-1}$. Los cambios en la composición iónica del agua y en la productividad primaria fueron igualmente acusados (SÁNCHEZ et AL., 2008). Las poblaciones de aves acuáticas, caracterizadas por el tarro blanco, la avoceta y el cuchara común en el periodo antecedente, como corresponde a lagunas salinas esteparias, fueron sustituidas por otras especies de más amplio espectro y aguas más dulces (fochas, malvasías, somormujos lavancos, porrones europeos, etc.), aumentando su riqueza y abundancia (SÁNCHEZ et AL., 2008).

Figura 2. Evolución mensual de la precipitación $(\mathrm{mm})$ en el observatorio meteorológico SIAR de Herencia durante el periodo de estudio (enero 2007-diciembre 2010). Se indica línea de tendencia.

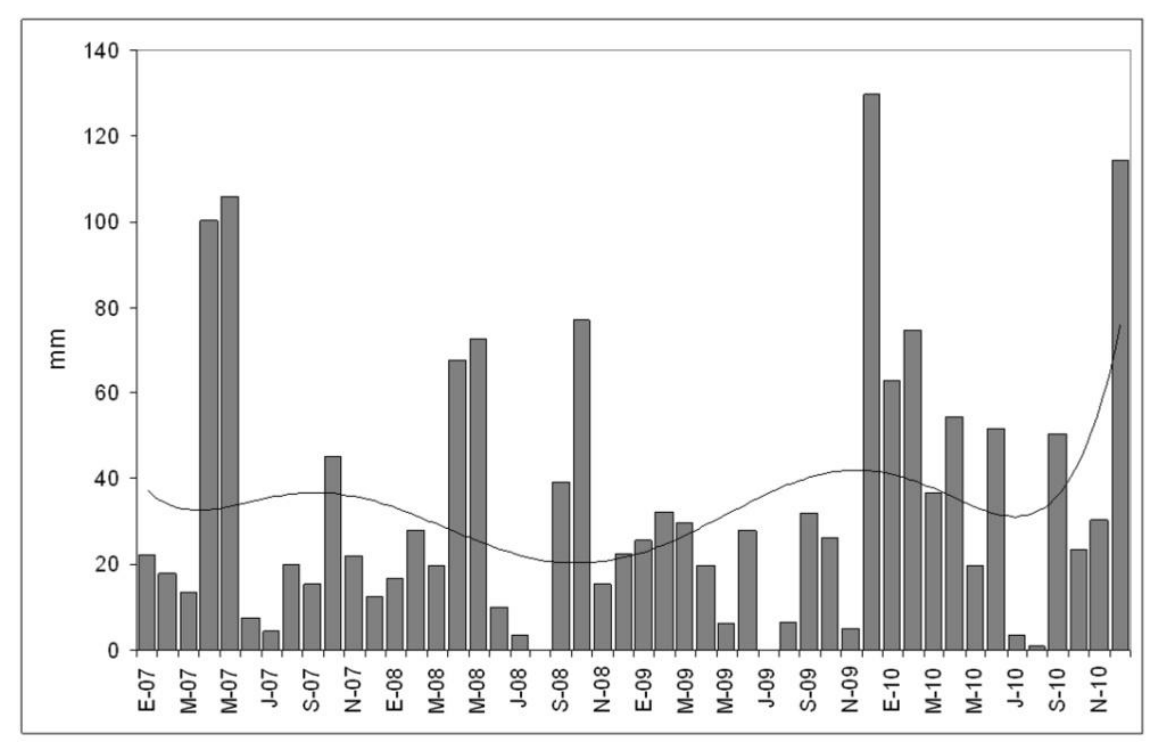

El otro aspecto a tener en cuenta a la hora de tipificar funcionalmente las lagunas estudiadas es analizar la evolución temporal del hidroperiodo, definido éste como la duración y la frecuencia de inundación de la cubeta (CMAJA, 2002).

El principal rasgo funcional de los sistemas acuáticos leníticos localizados en un contexto climático de carácter mediterráneo semiárido a árido, como es el caso de las lagunas de La Mancha Húmeda, es la variación anual e in- 
teranual del hidroperiodo, en consonancia con la precipitación. Estas variaciones se traducen en una alternancia de periodos de inundación y desecación, lo que hace que el funcionamiento de estos sistemas naturales sea definido de forma genérica como fluctuante (MARGALEF, 1987). Esta característica funcional, lejos de ser un obstáculo para la supervivencia de las lagunas, es su principal rasgo y valor, adaptándose los organismos vivos al mismo con diversas estrategias ecofisiológicas.

Para evaluar el hidroperiodo en las lagunas estudiadas se ha contabilizado si la cubeta permanecía seca o encharcada en cada visita mensual para cada localidad, a lo largo de los cuatro años de seguimiento. Los resultados del mismo se exponen en el CUADRO 2.

Como se puede comprobar, se pasa de una situación en la que prácticamente la totalidad de las lagunas permanecen con las cubetas encharcadas en el año 2007, a una situación de desecación de muchas de ellas en el verano de 2008, periodo seco que se amplía a finales de primavera y comienzos del otoño del año 2009, para finalmente recuperar un amplio periodo de encharcamiento en el año 2010. Cabe señalar como la laguna de Salicor ha permanecido encharcada de manera continua desde la afección de la tormenta "supercélula" en mayo de 2007 hasta agosto de 2009.

Cuadro 2. Periodos de inundación de las lagunas estudiadas entre los años 2007 a 2010. Resolución mensual (cuadro gris: laguna con agua; cuadro blanco: laguna seca).

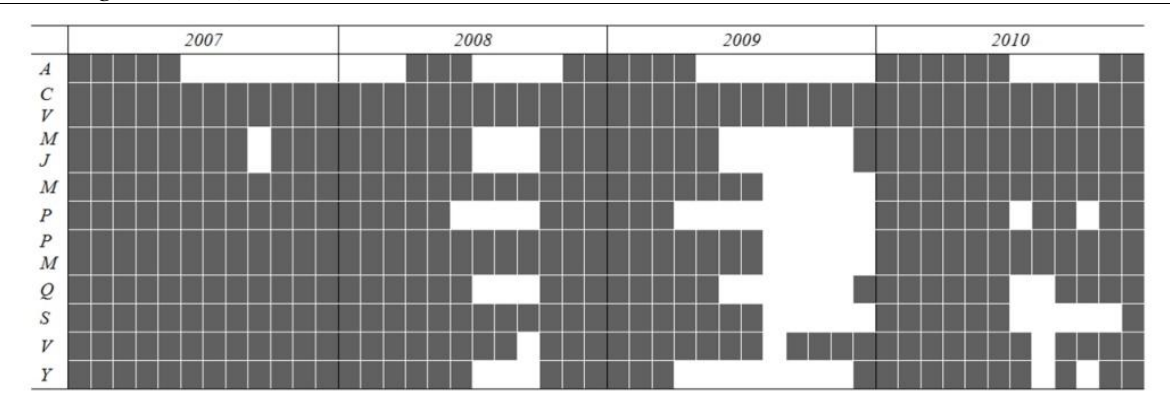

A=Alcahozo; $\mathrm{CV}=$ Camino de Villafranca; MJ=Manjavacas; $\mathrm{M}=$ Mermejuela; $\mathrm{P}=\mathrm{Pajares}$; PM=Pedro Muñoz; Q=Quero; $S=$ Salicor; V=Veguilla; $Y=$ Yeguas.

FUENTE: Datos propios.

\section{MÉTODOS}

\section{III.1. Especies objetivo.}

Las especies objeto de seguimiento son todas las aves acuáticas que gozan de un estado de conservación como SPEC 1 y SPEC 2 atendiendo a los crite- 
rios de Birds in Europe (Birdlife Internacional, 2004). Una especie se considera como SPEC 1 si se encuentra clasificada a nivel mundial en las categorías de "en peligro crítico", "en peligro", "vulnerable", "casi amenazada" o "insuficientemente conocida" bajo los criterios de la Lista Roja de la UICN (BAILLIE et AL., 2004). Una especie se considera como SPEC 2 si su población mundial se halla concentrada en Europa (más del 50\% de su población mundial o más del $50 \%$ de su área de distribución se incluye en Europa) y su estatus de conservación es desfavorable en este continente atendiendo a los criterios de la Lista Roja de la UICN o a los criterios adicionales desarrollados por TUCKER y HEATH (1994).

\section{III.2. Recogida y toma de datos en campo.}

La metodología seguida para la obtención de los datos se basa en un censo de dos tiempos (TELLERÍA, 1986), lo que nos ofrece cifras absolutas cuya fiabilidad se fundamenta en el gregarismo y relativa estabilidad en el espacio de las aves acuáticas (BERNIS, 1972). Los censos tuvieron una resolución temporal mensual, realizándose entre los meses de enero de 2007 y diciembre de 2010, con la excepción de los meses de enero de 2009 y agosto de 2010 en los que no se visitaron las lagunas.

La identificación de especies y el recuento de individuos se realizó siguiendo una estrategia múltiple en función de cómo se presentaban las aves en la lagunas. En la mayor parte de las ocasiones, cuando las especies se distribuían de manera dispersa, se procedió al conteo de cada individuo o conjunto de individuos de cada especie contenido en el campo visual del telescopio. Este conteo se realizó mediante el barrido de la laguna con la óptica mencionada (ej: 20 porrones europeos, 7 malvasías cabeciblancas, 5 agujas colinegras, etc.).

Cuando alguna especie se presentaba muy agrupada, lo que dificultaba el conteo individual, se optó por realizar un conteo por el método de bloques homogéneos, método que consiste en el recuento de un grupo o bloque de aves, que luego es extrapolado al conjunto. Estos bloques pueden ser de 10, 20, 30, 50 o 100 aves, dependiendo del tamaño del bando de aves a contabilizar. Este grupo de aves es usado como unidad de referencia para medir al bando, por ejemplo, un bloque o grupo de 20 aves puede ser usado para medir un bando de 100 aves en 5 grupos de 20 aves. Indicar que cuando se ha procedido de esta manera se aplicaba a una única especie.

Hay que señalar que algunas especies han sido infravaloradas por tener costumbres menos gregarias, por desarrollar gran parte de su actividad diaria entre la vegetación helofítica o por la dificultad de su detección e identifica- 
ción. Este último caso es el de los limícolas cuyas poblaciones reales pueden estar por encima de las ofrecidas en los conteos.

La observación con la instrumentación óptica adecuada, telescopio monocular de 20-60x65 y telescopio binocular de 20-60x80, se realizó desde uno o varios puestos dominantes en altura para cada laguna, procurando que existiera una meteorología favorable (viento flojo, temperaturas adecuadas, condiciones de luz adecuadas, sin precipitaciones fuertes y continuas).

Se procuró atender siempre a unos mismos criterios, al tiempo que mantener un esfuerzo similar de conteo con el fin de hacer comparables los datos obtenidos. A ello ha contribuido que los censos los ha realizado siempre dos equipos, uno de la UCLM y otro de la Universitat de València, cuyos coordinadores han sido siempre las mismas personas. Para evitar errores en el censo, en los casos que se consideró necesario, se hicieron varios recuentos y se tomó como válida la cifra más alta de las obtenidas.

Los resultados de los conteos de aves fueron anotados en el campo en un cuaderno o se grabaron in situ mediante el empleo de una grabadora magnética de sonidos, almacenándose posteriormente en una hoja de cálculo de MS Excel.

\section{III.3. Análisis de los datos.}

a) Caracterización de la abundancia y de la distribución espacial.

Se ha abordado la caracterización de la abundancia de las distintas especies a partir del establecimiento de cuatro categorías teniendo en cuenta para ello los datos obtenidos en los censos realizados en el periodo temporal de estudio:

$\checkmark$ Abundante: Especie que a lo largo del periodo de seguimiento ha presentado habitualmente cifras de más de 1.000 individuos.

$\checkmark$ Común: Especie que a lo largo del periodo de seguimiento ha presentado habitualmente cifras comprendidas entre 101 y 1.000 individuos.

$\checkmark$ Escasa: Especie que a lo largo del periodo de seguimiento ha presentado habitualmente cifras comprendidas entre 11 y 100 individuos.

$\checkmark$ Muy Escasa: Especie que a lo largo del periodo de seguimiento ha presentado habitualmente cifras comprendidas entre 1 y 10 individuos.

La caracterización de la distribución en el espacio de los distintos taxones estudiados se ha determinado a partir del establecimiento de tres categorías, establecidas a partir de los datos obtenidos en los censos realizados en el periodo analizado, teniendo en cuenta para ello el número de localidades en 
donde se ha registrado la presencia de las distintas especies. Así, tenemos las siguientes categorías:

$\checkmark$ Amplia: Especie que se ha localizado entre 7 y 10 lagunas (70-100 \%).

$\checkmark$ Discontinua: Especie que se ha localizado entre 4 y 7 lagunas (40-70 $\%)$.

$\checkmark$ Aislada: Especie que se ha localizado en menos de 4 lagunas (<40\%).

b) Caracterización de la estancia y de la tendencia en el tiempo.

Por estancia se entiende a la permanencia en el área de estudio de las distintas especies registradas durante el tiempo que ha durado el seguimiento. A la hora de establecer las distintas categorías de estancia, se ha optado por tener en cuenta el número de meses (frecuencia de aparición) en que se registró la presencia de cada especie durante el seguimiento, teniendo en cuenta que son 46 los meses de observación. Por ello, se ha establecido una clasificación en tres categorías o intervalos cuya definición y amplitud se basa en la frecuencia de aparición en el ámbito temporal analizado:

$\checkmark$ Constante: Se trata de aquellas especies que fueron registradas más de 30 veces en el total de 46 visitas efectuadas en el periodo de seguimiento.

$\checkmark$ Temporal: Se trata de aquellas especies que fueron registradas entre 12 y 30 veces en el total de 46 visitas efectuadas en el periodo de seguimiento.

$\checkmark$ Ocasional: Se trata de aquellas especies que fueron registradas menos de 12 veces en el total de 46 visitas efectuadas en el periodo de seguimiento.

Otro análisis que se ha realizado en la dimensión temporal es la evolución de las poblaciones mediante su tendencia en el tiempo, ayudándonos para ello del programa MS Excel.

Tres son las posibilidades que se pueden dar en relación con la tendencia: que sea ascendente (el número de aves aumenta en el tiempo), que sea descendente (el número de aves disminuye en el tiempo) o que se comporte estacionariamente (el número de aves se mantiene estable en el tiempo), mientras que el recorrido de la misma puede ser una línea recta (modelos lineales) o una curva (modelos no lineales).

De los distintos métodos que se pueden emplear para determinar la tendencia de una serie temporal, se ha optado por la obtención de una recta de regresión mediante el uso de modelos lineales o no lineales (logarítmico, polinomial, potencial o exponencial). En este caso se han empleado todos ellos 
tomándose la decisión de quedarnos con aquel que muestra un coeficiente de determinación $\left(\mathrm{R}^{2}\right)$ más elevado (HIGUERAS, 2003). Hay que indicar que con este análisis solo se pretende ayudar en la lectura de la tendencia, en ningún caso se pretende obtener un modelo de dinámica de poblaciones y por ello no se presenta en el gráfico correspondiente la función obtenida, tan solo el coeficiente $\mathrm{R}^{2}$.

\section{RESULTADOS Y DISCUSIÓN}

\section{IV.1. Evaluación de las especies identificadas en el seguimiento.}

a) Especies comunes, ampliamente distribuidas y constantes en el tiempo.

\section{Porrón europeo Aythya ferina SPEC 2}

Especie residente, se ha observado en 42 de las 46 visitas mensuales realizadas, pero con grandes diferencias de contingentes de una época a otra. En la mayor parte de las visitas se han registrado más de 150 individuos, con picos máximos en septiembre (1.049 aves) y octubre ( 700 aves) de 2010 y junio (652 aves) y diciembre (539 aves) de 2007. Es más regular y común como invernante y en primavera y comienzo del periodo reproductor (marzomayo), fechas en que se registran las cifras máximas, mientras que los mínimos se dan en la época postnupcial (septiembre-octubre), salvo la excepción comentada de 2010. Este comportamiento se atribuye al acusado descenso en los niveles de agua que se producen en los meses estivales en las lagunas, aunque también se han descrito movimientos de aves para mancar fuera de la región (JIMÉNEZ et AL., 1992). Teniendo en cuenta que se trata de un pato buceador, cuando los niveles hídricos de las lagunas son elevados tiende a presentarse de manera continuada, incluso con importantes efectivos. Hay que señalar que cuando debe reproducirse prefiere zonas con aguas permanentes y profundas y abundante vegetación, mientras que como invernante prefiere zonas de aguas libres. Se ha localizado en todas las lagunas estudiadas excepto en la de Alcahozo, cuyos niveles de agua son muy someros, siempre por debajo de los $30 \mathrm{~cm}$, lo que impide el establecimiento de la especie. La tendencia que presenta en el periodo de seguimiento es cíclica, disminuyendo al final del mismo.

En Europa se estimaron 210.000-440.000 parejas reproductoras (Birdlife Internacional, 2004); en España la población reproductora se evaluó en 16.600 ejemplares en 2007 (PALOMINO y MolinA, 2009); en Castilla-La Mancha la población invernante presentó un valor promedio de 6.380 individuos en el periodo 1990-2001 (MARTí y DEL MORAL, 2003).

\section{Cigüeña blanca Ciconia ciconia SPEC 2}


Especie residente, se ha observado en 38 de las 46 visitas mensuales realizadas, presentando importantes concentraciones desde finales de verano y hasta comienzos del invierno. Aunque es muy común en la región, son escasos los nidos localizados en las lagunas estudiadas, lo que se debe a que obtiene gran parte de su alimentación y ubica la mayoría de sus nidos alejados de las lagunas, por lo que no puede considerarse estrictamente como un ave acuática (VELASCO, 2006). Desde 1998 se observa una tendencia al aumento de la especie en la región (LÓPEZ DE CARRIÓN y MENOR, 1998). Aunque se localiza en ocho de las diez lagunas estudiadas, faltando solo en las lagunas de Alcahozo y Mermejuela, los principales contingentes siempre se han observado en la Reserva Natural de las lagunas de Alcázar de San Juan, donde era considerada como escasa hasta finales de los años ochenta. La razón se encuentra en la existencia de un basurero cercano, instalado a comienzos de la década de 1990, donde se alimentan la mayoría de los ejemplares que nidifican y duermen en las lagunas alcazareñas y su entorno (VELASCO, 2006). En la laguna de la Veguilla se ha registrado un máximo de 985 aves en octubre de 2009, nidificando aquí en nidos que se ubican en postes artificiales para favorecer su reproducción y en postes de la luz localizados en el entorno de la laguna (VELASCO, 2006). La tendencia que presenta en el periodo de seguimiento es cíclica, con un aumento al final del mismo.

En Europa se estimaron 180.000-220.000 parejas reproductoras (BIRDLIFE INTERNATIONAL, 2004); en España se estimó una población nidificante en 2004 de 33.217 parejas reproductoras (MOLINA y DEL MORAL, 2005); en Castilla-La Mancha 2.315 parejas seguras en 2004, la mayoría localizadas en la provincia de Ciudad Real, con 1.493 de ellas (MOLINA y DEL MORAL, 2005).

\section{b) Especies escasas, distribuidas de manera discontinua y temporales.}

\section{Avefría europea Vanellus vanellus SPEC 2}

Presente todo el año, se ha observado en 41 de las 46 visitas mensuales realizadas, siendo más común en otoño e invierno, existiendo además una pequeña población nidificante (en torno a las 20 parejas reproductoras). En general no suele sobrepasar los 40 ejemplares en las lagunas estudiadas, resultando más irregular y con un descenso de sus poblaciones en el año 2010. Sus contingentes invernantes fluctúan según la dureza del invierno, llegando a ser muy abundante los años más fríos (JIMÉNEZ et $A L$., 1992), como en 2007 cuando se observaron 508 individuos. En esta época se asocia principalmente a cultivos, prados, baldíos y pastizales alejados de los humedales, por lo que las cantidades censadas en ellos no resultan representativas de su población invernal (VELASCO, 2003). Para nidificar también prefiere los pas- 
tizales y saladares perilagunares (VELASCO, 2006). Es la única especie que se ha observado en todas las lagunas analizadas, aunque resulta más común en la laguna de Manjavacas y en la Reserva Natural de las Lagunas de Alcázar de San Juan. La tendencia que presenta en el periodo de seguimiento es cíclica, disminuyendo al final del mismo.

Figura 3. Evolución mensual de las poblaciones de porrón europeo (Aythya ferina) y cigüeña blanca (Ciconia ciconia) en las lagunas de la "Reserva de la Biosfera de La Mancha Húmeda" (enero 2007-diciembre 2010). Se indica línea de tendencia hallada y el valor del coeficiente de determinación $\left(r^{2}\right)$ obtenido.
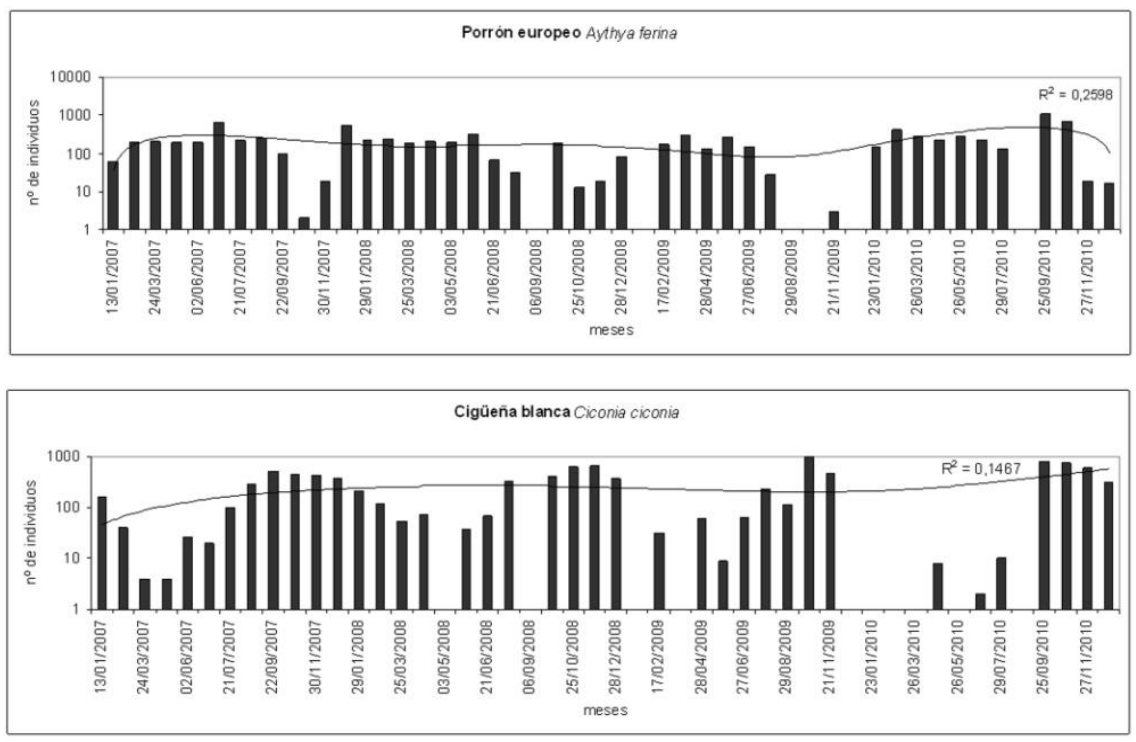

En Europa se estiman 1.700.000-2.800.000 parejas reproductoras (BIRDLIFE INTERNACIONAL, 2004); en España la población reproductora ha fluctuado entre 30-200 parejas y en Castilla-La Mancha se han estimado 497 parejas reproductoras (PÉREZ-ARANDA y MATUTE, 2003:255).

\section{Aguja colinegra Limosa limosa SPEC 2}

Migrante habitual que puede observarse durante todo el año, aunque no suele sobrepasar los 40 individuos, presentando pasos más conspicuos en primavera y otoño. Las observaciones estivales no son raras, con entrada de aves migratorias ya en julio-agosto en un paso que dura hasta octubre. Los máximos contingentes se han registrado precisamente en los meses estivales, con 531 aves en junio y 309 en julio de 2008, 265 individuos en agosto de 2009 y 212 aves en julio de 2007. Esta especie se ha observado en todas las lagunas analizadas excepto la laguna de Alcahozo. Presenta una tendencia 
cíclica en el periodo de seguimiento, con un aumento al final del mismo. Llama la atención que es más regular y presenta mayores efectivos en los años 2007 y 2008, volviéndose más raro en los años 2009 y 2010.

En Europa se estimaron 130.000-240.000 parejas reproductoras (BIRDLIFE INTERNATIONAL, 2004); en España la población nidificante se ha cifrado en unas 4-10 parejas reproductoras (HORTAS, 2004), aunque en el año 2007 no se detectó su reproducción en nuestro país (PALOMINO y MOLINA, 2009:127), siendo la población invernante muy abundante y hallándose muy concentrada en Andalucía occidental (MARTí y DEL MORAL, 2003); en Castilla-La Mancha se ha comportado como un reproductor esporádico (HORTAS, 2004). La intensificación de la actividad agraria y el drenaje y desecación de lagunas han provocado la reducción del hábitat que sustentan sus poblaciones (HORTAS, 2004).

\section{Archibebe común Tringa totanus SPEC 2}

Especie que puede observarse en cualquier momento del año, pero sobre todo está presente en los pasos migratorios, especialmente en el primaveral aunque tampoco falta en el otoñal. No suele sobrepasar los 40 individuos, pero en el paso primaveral de 2008 se llegaron a observar 265 aves (abril). Como invernante es muy escaso e irregular. Presenta una reducida población nidificante en la las lagunas de Alcázar de San Juan y en la laguna de Manjavacas. Su estimación se ve dificultada en la época reproductora pues coincide con el paso migratorio primaveral de las poblaciones que crían en el norte de Europa, coincidiendo aves reproductoras (muy escasas) y migrantes (muy abundantes) (VELASCO y MolinA, 2003). No presenta una tendencia clara en el periodo de seguimiento, pero al igual que sucede con otros limícolas, llama la atención que es más regular y presenta mayores efectivos hasta la primera mitad del año 2009 , volviéndose más raro a partir de ese momento. Esta especie se ha observado en todas las lagunas analizadas excepto la laguna de Pajares, aunque es la laguna de Manjavacas la que se ha revelado como la más importante.

En Europa se han estimado 280.000-610.000 parejas reproductoras (BIRDLIFE INTERNACIONAL, 2004); en España la población nidificante en 2007 se evaluó en 5.600 individuos (PALOMINO y MolinA, 2009); en Castilla-La Mancha la población reproductora probablemente no supera las 30-50 parejas (JIMÉNEZ et AL., 1992; HORTAS et AL., 2003), concentrándose en la laguna de Manjavacas un $\mathrm{n}^{\circ}$ importante de reproductores (PALOMINO y MolinA, 2009). En las lagunas de Alcázar de San Juan también cría con bastante regularidad dentro de su escasez (VELASCO, 2006). La pérdida y transformación del hábitat y la depredación y molestias durante la reproduc- 
ción ha provocado la reducción del hábitat que sustentan sus poblaciones (HORTAS et AL., 2004).

\section{Combatiente Philomachus pugnax SPEC 2}

Migrador habitual, sobre todo en el paso primaveral, momento en el que se registran las cifras máximas (119 aves en abril de 2008, 86 aves en abril de 2007). El paso otoñal presenta máximos en los meses de septiembre y octubre. Existe también un contingente invernante más reducido, que no supera los 20 individuos. Presenta una tendencia cíclica en el periodo de seguimiento, con un descenso al final del mismo. Es más regular y presenta mayores efectivos en los años 2007 a 2009, volviéndose más raro en el año 2010. Se ha observado su presencia en siete de las diez lagunas analizadas, faltando en las lagunas de Alcahozo, Mermejuela y Pajares.

En Europa se han estimado 200.000-510.000 parejas reproductoras (BIRDLIFE INTERNACIONAL, 2004); en España no se reproduce la especie, estimándose la población invernante en 260-3.000 individuos (BIRDLIFE INTERNACIONAL, 2004); En Castilla-La Mancha la población media inver nante en el periodo 1990-2001 ha sido de 270 aves (MARTí y DEL MORAL, 2003).

c) Especies muy escasas, de distribución restringida y ocasionales en el tiempo.

\section{Cigüeña negra Ciconia nigra SPEC 2}

Especie ocasional, de fenología migratoria, que solo ha sido observada en dos ocasiones siempre en el paso migratorio postnupcial (septiembre de 2007 y 2008), apareciendo en pequeños grupos de menos de diez individuos. MADROÑO et AL. (1992) indican que en la mayor parte de los casos los individuos que se observan en estas fechas son aves no ibéricas. Seguramente la especie aparece todos los años de manera habitual pero al no quedarse los ejemplares mucho tiempo en una laguna pueden pasar desapercibidos (VelasCO, 2006). Se ha observado en la laguna de la Veguilla y en Pedro Muñoz.

En Europa se estimaron 7.800-12.000 parejas reproductoras (BIRDLIFE INTERNACIONAL, 2004); en España la población nidificante se ha evaluado en 387 parejas reproductoras (SEO/BIRDLIFE, 2002); en Castilla-La Mancha se ha constatado la presencia de 24 parejas, la mayoría (14 parejas) localizadas en el SW de la provincia de Ciudad Real (CANO y HERNÁNDEZ, 2003). Su población en España se ha ido incrementando desde el censo nacional de 1987, aunque parte de este aumento se debe a un mejor seguimiento de la 
Figura 4. Evolución mensual de las poblaciones de avefría europea (Vanellus vanellus), aguja colinegra (Limosa limosa), archibebe común (Tringa totanus) y combatiente (Philomachus pugnax) en las lagunas de la Reserva de la Biosfera de La Mancha Húmeda" (enero 2007-diciembre 2010). Se indica línea de tendencia hallada y el valor del coeficiente de determinación $\left(r^{2}\right)$ obtenido.
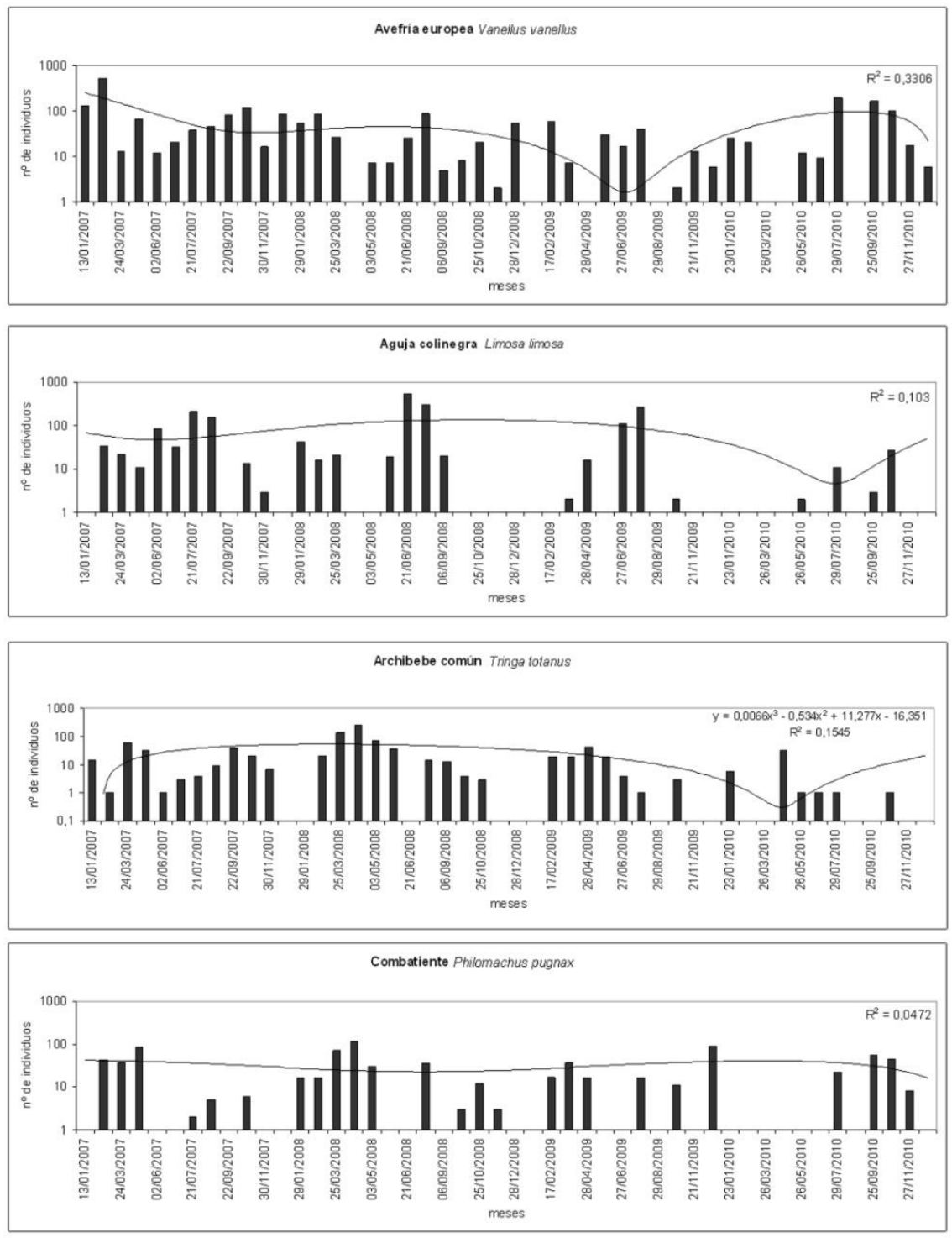

especie (CANO y HERNÁNDEZ, 2004). Molestias humanas producidas por actividades forestales y recreativas, colisiones y electrocuciones con tendidos 
eléctricos y la contaminación de los ecosistemas acuáticos que sustentan a la especie son las principales amenazas que se ciernen sobre ella (CANO y HERNÁNDEZ, 2004). Castilla-La Mancha cuenta con un Plan de Recuperación aprobado en el año 2003 (DECRETO 275/2003, 9 de septiembre).

\section{Espátula común Platalea leucorodia SPEC 2}

Migrante ocasional, con casi todas las citas en el paso postnupcial. 25 espátulas se observan en el mes de julio de 2010 en la Reserva Natural de Las Lagunas de Alcázar de San Juan (Camino de Villafranca y Veguilla), tres en septiembre de 2007 en la laguna de la Veguilla, dos en el mes de abril de 2009 en la laguna de Pedro Muñoz y una en octubre de 2007 de nuevo en la laguna de la Veguilla.

En Europa se estimaron 8.900-15.000 parejas reproductoras a principios de la década de 2000 (Birdlife Internacional, 2004); en España se alcanzó una población reproductora de 1.614 parejas reproductoras en 2007 (MÁÑEZ y RENDÓN-MARTOS, 2009); en Castilla-La Mancha se produjo en el año 2005 un intento fallido de cría de tres parejas en el embalse de la Vega del Jabalón (Ciudad Real), así como una reproducción exitosa en el embalse de Azután (Toledo) que sacó adelante dos pollos (Velasco et AL., 2007). Molestias humanas y de perros asilvestrados, la presión urbanística, la contaminación de los ecosistemas acuáticos y la concentración de la población invernal en Mauritania son los principales factores de amenaza que afectan a la especie (DE LE COURT et AL., 2004).

\section{Cerceta pardilla Marmaronetta angustirostris SPEC 1}

Especie muy escasa, con citas esporádicas en ambos pasos migratorios relacionadas con los movimientos oportunistas que suele realizar la especie. Se ha observado un ejemplar en la laguna de Salicor en el mes de octubre de 2007 y otro en la laguna de la Veguilla en el mes de marzo de 2010. Antiguo nidificante, se reprodujo por última vez en las lagunas de Alcázar de San Juan en 1970 (JIMÉNEZ et AL., 1992).

En Europa se estiman 390-1.000 parejas reproductoras (BIRDLIFE INternacional, 2004); en España la población nidificante no supera las 200 parejas (GREEN et AL., 2003), evaluándose en el año 2007 en 96 parejas reproductoras que sacaron adelante 332 pollos (BALLESTEROS et AL., 2008), siendo la población invernante muy escasa y con una población muy concentrada; en Castilla-La Mancha una pareja reproductora en la laguna de Pétrola (Albacete) en 1998 (GREEN et AL., 2003). La degradación de los humedales someros y estacionales, la caza furtiva y las interacciones con otras especies (cangrejo rojo, el flamenco y el calamón) son las principales amenazas que ponen en 
Figura 5. Evolución mensual de las poblaciones de cigüeña negra (Ciconia nigra), espátula común (Platalea leucorodia), cerceta pardilla (Marmaronetta angustirostris) y zarapito real (Numenius arquata) en las lagunas de la Reserva de la Biosfera de La Mancha Húmeda” (enero 2007-diciembre 2010).
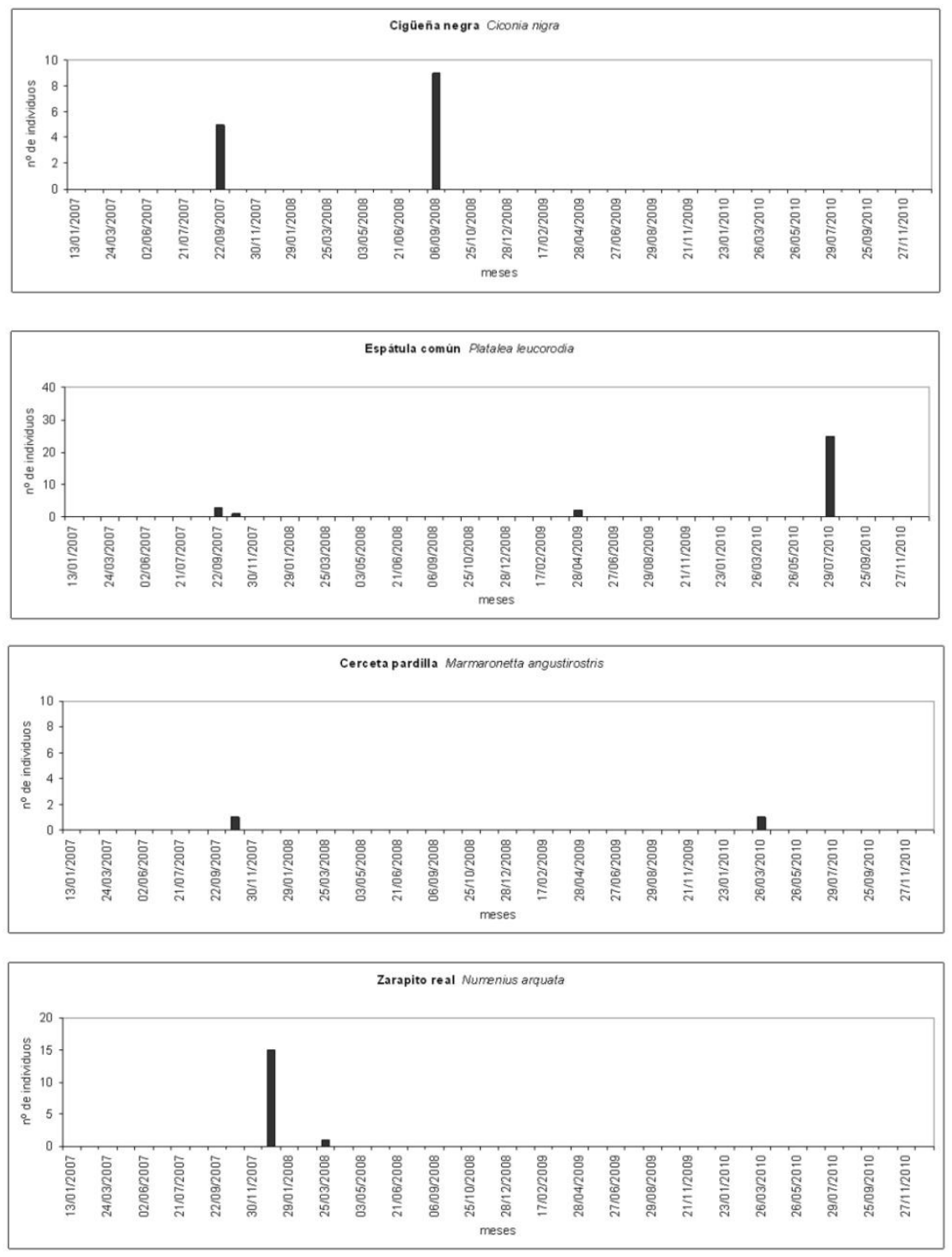

peligro su supervivencia a largo plazo (GREEN et AL., 2004). La existencia de movimientos entre España y el norte de África sugiere la incorporación de efectivos de la población africana a la española, lo que podría estar ocul- 
tando un declive aún mayor de nuestras poblaciones, que estarían actuando como sumideros demográficos (GREEN et AL., 2002).

\section{Zarapito real Numenius arquata SPEC 2}

Calificada antaño como un ave frecuente en los pasos migratorios, ahora es muy rara y escasa. Solo se han observado 15 individuos en el mes de diciembre de 2007 en la laguna de Yeguas y otra ave en el mes de marzo de 2008 en la laguna Grande de Quero.

En Europa se estiman 183.000-214.500 parejas reproductoras (THORUP, 2006); en España la población nidificante en 2007 y 2008 ha sido de 3 parejas localizadas todas ellas en Galicia (PALOMINO y MolinA, 2009). En Castilla-La Mancha no se ha podido constatar la reproducción reciente de la especie, aunque existe una cita de la primavera de 1991 en la laguna de Camino de Villafranca donde se escucharon cantos de celo y se vieron dos ejemplares en abril y julio (GULLICK y JIMÉNEZ, 1991) que sugieren un posible evento de cría (PALOMINO y MolinA, 2009). La destrucción de los hábitats que sustentan a la especie (por forestación, intensificación agrícola, pérdida de áreas de alimentación y por la actividad ganadera) y las molestias humanas y de perros asilvestrados amenaza la supervivencia de la especie a corto plazo.

\section{d) Otras especies.}

\section{Malvasía cabeciblanca Oxyura leucocephala SPEC 1}

La presencia de esta especie en las lagunas de La Mancha Húmeda comenzó a ser frecuente a partir de 1988, considerándose accidental con anterioridad (VELASCO, 2006). Hoy se puede considerar como una especie residente y reproductora en la zona, constatándose la primera nidificación comprobada para Castilla-La Mancha en la laguna de Pedro Muñoz en 1990 (tres polladas con un total de 11 pollos) (JiMÉNEZ et $A L$., 1992). La población ha variado entre 20 y 90 individuos en la mayor parte de las visitas realizadas. Su presencia coincide con niveles hídricos elevados, que en la actualidad se producen de manera continuada en las lagunas que reciben vertidos de aguas residuales depuradas (Manjavacas, Pedro Muñoz y La Veguilla). La dinámica anual de la malvasía cabeciblanca es típicamente de una especie constante y reproductora, presentando cifras bajas en el periodo invernal, donde llega a desaparecer, y máximos en los meses de marzo y junio, observándose hasta 148 individuos en el mes de marzo de 2010 o 136 aves en el mes de julio de 2008. No se ha observado en las lagunas de Alcahozo, Yeguas, Mermejuela y Grande de Quero. Manjavacas, Salicor, Pedro Muñoz y Veguilla son las lagunas más importantes para la especie. 
En Europa se ha estimado una población reproductora de 550-1.400 parejas (BIRDLIFE INTERNACIONAL, 2004) y una población invernante superior a los 5.700 individuos; en España en 2007 sus efectivos se cifraron en la época reproductora en unos 1.003-1.058 ejemplares (BALLESTEROS et AL., 2008), presentando una población invernante escasa y concentrada en Andalucía y la Comunidad Valenciana (El Hondo); en Castilla-La Mancha en el año 2007 se registraron 346-357 individuos durante la época de reproducción (BALLESTEROS et AL., 2008). La actividad cinegética, la destrucción y la degradación del hábitat y la expansión de la malvasía canela son factores que amenazan la supervivencia futura de la especie (TORRES ESQUIVIAS, 2004). Junto a la cigüeña negra, esta especie cuenta también con un Plan de Recuperación en la comunidad autónoma, aprobado en 1995.

Figura 6. Evolución mensual de las poblaciones de malvasía cabeciblanca (Oxyura leucocephala) y grulla común (Grus grus) en las lagunas de la Reserva de la Biosfera de La Mancha Húmeda" (enero 2007-diciembre 2010). Se indica línea de tendencia hallada y el valor del coeficiente de determinación $\left(r^{2}\right)$ obtenido.
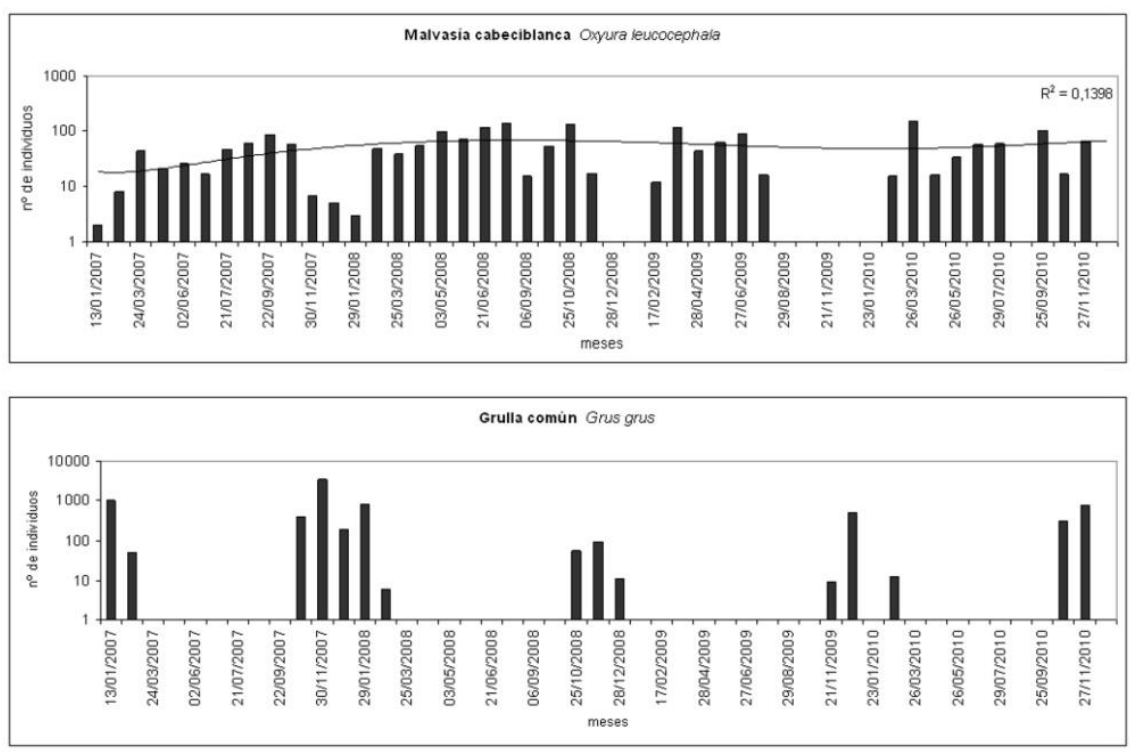

Grulla común Grus grus SPEC 2

Especie típicamente invernante, con un contingente variable (94 individuos en 2008 y más de 3.000 en 2007) en función de los niveles hídricos, la disponibilidad de alimento, las molestias humanas y los rigores climáticos (VELASCO, 2003). La especie comienza a observarse en la zona a partir del 
mes de octubre, abandonándola en el mes de marzo, acumulándose el mayor número de observaciones en los meses de noviembre y diciembre. El núcleo más importante para la invernada de la grulla en Castilla-La Mancha lo conforma La Mancha húmeda, con unas 13.000 aves, ocupando numerosos cultivos entorno a las Tablas de Daimiel y varias de las lagunas estudiadas que son utilizadas como dormidero (Manjavacas, Mermejuela, Pajares y Salicor) (PRIETA y DEL MORAL, 2008). También utiliza estas lagunas en el paso migratorio, siendo más rápido y notorio en primavera que en otoño. La especie es visible en vuelo por toda la región en los meses de otoño e invierno.

En el año 2000 se estimaron en Europa 90.250 parejas reproductoras (BIRDLIFE INTERNACIONAL, 2004), ascendiendo la población invernante en el año 2007 a 218.220 individuos (PRIETA y DEL MORAL, 2008:); en España los efectivos invernantes se cifraron en la temporada 2007-08 en unos 151.423 ejemplares (PRIETA y DEL MORAL, 2008); en Castilla-La Mancha la población invernante en esa misma temporada 2007-08 ascendió a 27.504 individuos (PRIETA y DEL MORAL, 2008).

\section{IV.2. Valoración de cada laguna estudiada.}

En el CUADRO 3 se muestra la distribución espacial en las lagunas estudiadas de las especies de aves acuáticas amenazadas atendiendo a los criterios de Birds in Europe sometidas a seguimiento durante el periodo 2007-2010, cuadro que nos sirve de base para abordar el análisis valorativo de cada una de ellas, junto con los resultados numéricos para cada especie.

\section{a) Reserva Natural Complejo lagunar de Alcázar de San Juan (Ciudad Real).}

Conjunto de tres lagunas (La Veguilla, Camino de Villafranca y Las Yeguas) localizadas al oeste del núcleo urbano de Alcázar de San Juan que suman un total de 342 ha de extensión superficial. La profundidad máxima ha llegado a los $150 \mathrm{~cm}$ como consecuencia de la tormenta "supercélula" que afectó a la zona en mayo de 2007, pero la profundidad habitual no supera el metro. Se trata de lagunas de aguas hiposalinas a hipersalinas (Yeguas), de tipo iónico mixto sulfatado clorurado-magnésico cálcico, dominando los helófitos (carrizo Phragmites australis, enea Typha dominguensis, junco de laguna Schoenoplectus lacustris y castañuela Bolboschoenus maritimus) solamente en la laguna de La Veguilla como consecuencia de los aportes de aguas residuales que recibe. También existen formaciones de tarayes. La presencia de playas para limícolas es variable, sufriendo un importante deterioro en los últimos años la laguna del Camino de Villafranca por erosión del agua favorecida por el viento (oleaje). Su declaración como Reserva Natural 
se produjo en el mes de octubre de 1999. La laguna de la Veguilla es la que ha albergado un mayor número de especies objetivo, faltando tan solo el zarapito real Numenius arquata, así como la localidad que ha recogido un mayor número de individuos de todas las lagunas estudiadas, 11.989 aves. Le sigue en importancia la laguna del Camino de Villafranca, con 9 especies y 4.714 aves, y la laguna de Yeguas, con 7 especies y 993 individuos. Este complejo de lagunas es de importancia para la cigüeña blanca (las tres lagunas) y el porrón europeo (Veguilla y Camino de Villafranca) y en menor medida para la malvasía cabeciblanca (Veguilla) y la aguja colinegra (Camino de Villafranca).

Cuadro 3. Especies de aves clasificadas como SPEC 1 y 2 y su distribución en lagunas de la Reserva de la Biosfera de "La Mancha Húmeda" (enero-2007/diciembre-2010).

\begin{tabular}{|c|c|c|c|c|c|c|c|c|c|c|c|c|}
\hline & $S P E C$ & $A$ & $\mathrm{CV}$ & $M J$ & $M R$ & $P$ & $P M$ & $Q$ & $S$ & $V$ & $Y$ & $N^{\circ}$ lagunas \\
\hline Marmaronetta angustirostris & 1 & 0 & 0 & 0 & 0 & 0 & 0 & 0 & 1 & 1 & 0 & 2 \\
\hline Aythya ferina & 2 & 0 & 1 & 1 & 1 & 1 & 1 & 1 & 1 & 1 & 1 & 9 \\
\hline Oxyura leucocephala & 1 & 0 & 1 & 1 & 0 & 1 & 1 & 0 & 1 & 1 & 0 & 6 \\
\hline Ciconia ciconia & 2 & 0 & 1 & 1 & 0 & 1 & 1 & 1 & 1 & 1 & 1 & 8 \\
\hline Ciconia nigra & 2 & 0 & 0 & 0 & 0 & 0 & 1 & 0 & 0 & 1 & 0 & 2 \\
\hline Platalea leucorodia & 2 & 0 & 1 & 0 & 0 & 0 & 1 & 0 & 0 & 1 & 0 & 3 \\
\hline Grus grus & 2 & 0 & 1 & 1 & 1 & 1 & 0 & 0 & 1 & 1 & 0 & 6 \\
\hline Vanellus vanellus & 2 & 1 & 1 & 1 & 1 & 1 & 1 & 1 & 1 & 1 & 1 & 10 \\
\hline Philomachus pugnax & 2 & 0 & 1 & 1 & 0 & 0 & 1 & 1 & 1 & 1 & 1 & 7 \\
\hline Limosa limosa & 2 & 0 & 1 & 1 & 1 & 1 & 1 & 1 & 1 & 1 & 1 & 9 \\
\hline Numenius arquata & 2 & 0 & 0 & 0 & 0 & 0 & 0 & 1 & 0 & 0 & 1 & 2 \\
\hline Tringa totamus & 2 & 1 & 1 & 1 & 1 & 0 & 1 & 1 & 1 & 1 & 1 & 9 \\
\hline$N^{\circ}$ especies & & 2 & 9 & 8 & 5 & 6 & 9 & 7 & 9 & 11 & 7 & \\
\hline
\end{tabular}

A=Alcahozo; $\mathrm{CV}=$ Camino de Villafranca; $\mathrm{MJ}=$ Manjavacas; MR=Mermejuela; $\mathrm{P}=$ Pajares; PM=Pedro Muñoz; Q=Quero; S=Salicor; V=Veguilla; Y=Yeguas. / O=Ausencia especie; 1=Presencia especie

\section{b) Reserva Natural de la Laguna de Salicor (Ciudad Real).}

Laguna localizada al norte del núcleo urbano de Campo de Criptana, cuya superficie asciende a un total de 57 ha, alcanzando una profundidad máxima de $200 \mathrm{~cm}$ como consecuencia de la tormenta "supercélula" que afectó a la zona en mayo de 2007, pero la profundidad habitual no supera el medio metro. Sus aguas han sido hiposalinas durante el periodo de seguimiento por esta misma razón, aunque habitualmente es hipersalina, con tipo iónico mixto sulfatado-magnésico cálcico. La presencia de helófitos es testimonial, si hay tarayes, siendo importante la extensión que ocupan las playas para las aves limícolas. Su declaración como Reserva Natural se produjo en el mes de enero de 2001. La laguna ha albergado en el periodo de seguimiento nueve de las doce especies objetivo observadas, sumando un total de 2.304 aves. Esta laguna es de importancia para el porrón europeo y la grulla común, manteniendo esta última especie un importante dormidero y lugar de alimentación en su entorno. 


\section{c) Reserva Natural del Complejo lagunar de Pedro Muñoz (Ciudad Real).}

Integrada por dos lagunas, la de Retamar y la del Pueblo o Pedro Muñoz, nosotros sólo hemos estudiado esta segunda. Se localiza inmediatamente al norte del núcleo urbano de Pedro Muñoz, ascendiendo su superficie a 36 ha, presentando una profundidad máxima de $80 \mathrm{~cm}$. Sus aguas son hiposalinas, de tipo iónico mixto sulfatado clorurado-magnésico cálcico. La presencia de helófitos es muy relevante, tanto en la orilla como en la propia lámina de agua abierta, dominando el carrizo Phragmites australis, la enea Typha dominguensis, el junco de laguna Schoenoplectus lacustris y la castañuela Bolboschoenus maritimus. También hay rodales y manchas perilagunares de tarayes. La extensión que ocupan las playas para limícolas no es muy importante, aunque las hay. Su declaración como Reserva Natural se produjo en el mes de octubre de 2002. La laguna ha albergado en el periodo de seguimiento nueve de las doce especies objetivo observadas, sumando un total de 2.511 aves. Es la principal localidad para la supervivencia de la malvasía cabeciblanca de todas las estudiadas, siendo también un lugar de importancia para el porrón europeo.

\section{d) Reserva Natural del Complejo lagunar de Manjavacas (Cuenca-Ciudad Real)}

Conjunto de cuatro lagunas localizadas al este del núcleo urbano de Pedro Muñoz (Ciudad Real) y al sur del de Mota del Cuervo (Cuenca), tratándose de las lagunas de Manjavacas, Alcahozo, La Dehesilla y la laguna de Sánchez Gómez. En el programa de seguimiento solo se han incluido las dos primeras, sumando un total de 300 ha de extensión superficial, con una profundidad máxima que no ha superado los $100 \mathrm{~cm}$. Como consecuencia de los vertidos de aguas residuales, la laguna de Manjavacas es hiposalina y de tipo iónico bicarbonato clorurado-magnésico sódico, mientras que la laguna de Alcahozo es hipersalina y de tipo iónico sulfatado clorurado-magnésico sódico. Los helófitos solamente dominan en la laguna de Manjavacas, apareciendo el carrizo Phragmites australis, la enea Typha dominguensis, el junco de laguna Schoenoplectus lacustris y la castañuela Bolboschoenus maritimus. Las playas para las aves limícolas son extensas, favoreciendo la presencia de los mismos. Su declaración como Reserva Natural se produjo en el mes de octubre de 2001. La laguna de Manjavacas ha albergado un buen número de especies objetivo, hasta ocho taxones, con un total de 7.431 aves, mientras que la laguna de Alcahozo es la localidad en la que se ha observado un menor número de especies de todas las lagunas estudiadas, con tan solo dos (avefría Vanellus vanellus y archibebe común Tringa totanus) y 14 aves registradas. La laguna de Manjavacas es una localidad de interés para el porrón europeo, la avefría europea y la grulla común (cuenta con un importante 
dormidero). Por su parte, la laguna de Alcahozo solo sería de interés para el archibebe común y la avefría europea, las únicas especies que se han avistado en ella y no precisamente con grandes contingentes.

\section{e) Espacio Natural Protegido en Tramitación: Laguna Grande de Quero (Toledo)}

Aunque en el mes de noviembre de 2005 se sometió a tramite de información pública y de audiencia de los interesados el PORN de la Laguna Grande de Quero, hasta la fecha no se ha resuelto la declaración de la misma como espacio natural protegido. Intereses salineros y de aprovechamiento de sus aguas pueden haber ejercido presión para que la Junta de Comunidades haya dilatado durante tanto tiempo una toma de decisión en relación con su protección. En cualquier caso estamos ante una laguna con una superficie de 78 ha y una profundidad máxima de $200 \mathrm{~cm}$ como consecuencia de la acción de la tormenta supercélula del mes de mayo de 2007, dejando incomunicado el acceso al núcleo urbano de Quero desde la localidad de Villafranca de los Caballeros durante varias semanas. Precisamente la laguna se localiza aneja al pueblo por el SW. Sus aguas son hipersalinas, de tipo clorurado bicarbonatado sulfatado-potásico magnésico, lo que puede estar relacionado con el vertido de aguas residuales desde la depuradora existente en el núcleo urbano de Quero. No existen helófitos en la lámina de agua y prácticamente son anecdóticos en el cinturón perilagunar externo, apareciendo en este caso algunos rodales de juncos (Juncus sp. y junco churrero Scirpoides holoschoenus). La presencia de playas óptimas que pueden aprovechar las aves limícolas es importante, beneficiando a las mismas. La laguna ha albergado en el periodo de seguimiento siete de las doce especies objetivo observadas, cinco de ellas limícolas, sumando un total de 166 aves, siendo por ello la segunda laguna con un menor número de registros en cuanto a la abundancia. Localidad de importancia para el porrón europeo y la aguja colinegra.

\section{f) Lagunas sin protección: Mermejuela (Toledo) y Pajares (Ciudad Real).}

Se trata de las únicas dos lagunas que no gozan de protección como espacios naturales protegidos, aunque si son consideradas como zonas sensibles toda vez que forman parte de la Red Natura 2000 (LIC y ZEPA "Humedales de La Mancha"). La laguna de Mermejuela se localiza al oeste del núcleo urbano de Miguel Esteban, perteneciendo a este municipio, mientras que la laguna de Pajares se halla al norte del núcleo urbano de Alcázar de San Juan, incluyéndose en su municipio. Se trata de las dos lagunas de menor tamaño, 9 y 22 ha respectivamente, y que se encuentran más aisladas del conjunto de las estudiadas, lo que puede haber favorecido su exclusión como espacio natural protegido. Para la laguna de Mermejuela carecemos de datos hidroquí- 
micos, aunque sus aguas son salinas, mientras que la de Pajares es hipersalina y de tipo iónico mixto sulfatado clorurado-magnésico sódico. En ambos casos sus láminas de agua se encuentran libres de helófitos, siendo testimonial en el cinturón perilagunar (algunos rodales de juncos). La laguna de $\mathrm{Pa}$ jares dispone de playas extensas, todo lo contrario que la laguna de Mermejuela cuyas orillas se hallan vegetadas por pastizales halonitrófilos y presentando una cierta pendiente que no favorece a las aves limícolas. En Mermejuela se han detectado 5 especies y en Pajares 6 de las que se han sometido a seguimiento en este trabajo, observándose 364 aves en la primera laguna y 4.135 en la segunda. El elevado número de aves registradas en la laguna de Pajares se debe a la presencia de la grulla común que tiene aquí uno de sus mejores dormideros en la zona, al tiempo que todo el entorno de la laguna funciona como área de alimentación para la especie. Ambas localidades son de interés para la grulla común.

\section{CONCLUSIONES}

Doce de las quince especies clasificadas como SPEC 1 y SPEC 2 en España fueron observadas en las 10 lagunas seleccionadas de la Reserva de la Biosfera de La Mancha Húmeda.

Solo dos especies son comunes y se distribuyen ampliamente en estas lagunas, el porrón europeo Aythya ferina y la cigüeña blanca Ciconia ciconia, frente a cuatro especies (cigüeña negra Ciconia nigra, espátula común Platalea leucorodia, cerceta pardilla Marmaronetta angustirostris y zarapito real Numenius arquata) que fueron registradas ocasionalmente y con bajos números.

El porrón europeo Aythya ferina es la especie que muestra el mayor tamaño poblacional, tanto en los meses de invernada como de reproducción, sufriendo siempre un descenso demográfico importante después del periodo reproductor.

En relación con la malvasía cabeciblanca, la única especie catalogada como SPEC 1 con presencia regular en la zona, es la laguna de Pedro Muñoz la principal localidad para la supervivencia de la especie, teniendo también importancia las lagunas de la Veguilla, Manjavacas y, coyunturalmente, la de Salicor.

La grulla común Grus grus inverna en estas lagunas habitualmente, siendo cuatro de las localidades estudiadas de interés tanto por albergar importantes dormideros para la especie como por resultar su entorno una importante área de alimentación. Se trata de las lagunas de Salicor, Manjavacas, Pajares y Mermejuela. 
Cinco especies se reproducen habitualmente en las lagunas estudiadas (avefría europea Vanellus vanellus, malvasía cabeciblanca Oxyura leucocephala, porrón europeo Aythya ferina, cigüeña blanca Ciconia ciconia y archibebe común Tringa totanus).

El resto de especies presenta una fenología típica de migrantes usando estas lagunas como área de descanso en sus viajes migratorios. Hay que destacar, además, que la aguja colinegra Limosa limosa y la cigüeña blanca Ciconia ciconia aprovechan las lagunas estudiadas como área de concentración estival antes de la migración otoñal.

Son cinco las lagunas que durante el periodo de estudio registran un elevado número de especies (de 8 a 10) y una mayor abundancia, tratándose en todos los casos de lagunas que se encuentran protegidas legalmente como Reserva Natural (Camino de Villafranca, Manjavacas, Pedro Muñoz, Salicor y La Veguilla).

La laguna de la Veguilla es la localidad que ha albergado un mayor número de especies objetivo, así como la localidad que ha recogido un mayor número de individuos de todas los taxones estudiados, 11.989 aves. Le siguen en importancia las lagunas del Camino de Villafranca, con 9 especies y 4.714 aves, y la de Manjavacas con ocho especies y un total de 7.431 aves.

Las laguna de la Veguilla y Camino de Villafranca se han revelado, además, como las localidades que presentan una mayor importancia para la cigüeña blanca y el porrón europeo.

Las lagunas de Salicor y de la Veguilla son las únicas en las que se ha observado la presencia de la otra especie catalogada como SPEC 1, la cerceta pardilla Marmaronetta angustirostris.

La profundidad de la lámina de agua, la presencia de playas adecuadas en las orillas, el desarrollo de helófitos, la presencia de nutrientes y las características hidroquímicas de las lagunas parecen controlar el uso de este conjunto de lagunas por las diferentes especies.

\section{AGRADECIMIENTOS}

Este trabajo ha sido posible en parte gracias al soporte financiero de la Junta de Comunidades de Castilla-La Mancha (JCCM), a través de dos proyectos regionales de investigación PREG-05-16 y PAC06-0116.

\section{BIBLIOGRAFÍA}

Baillie, J.E.M.; Hilton-TAYlor, C.; Stuart, S.N., eds. (2004). 2004 IUCN Red 
List of Threatened Species ${ }^{\mathrm{TM}}$. A Global Species Assessment. IUCN, Gland, Switzerland and Cambridge, UK. En línea: http://data.iucn.org/dbtwwpd/commande/downpdf.aspx?id=10588\&url=http://www.iucn.org/dbtwwpd/edocs/RL-2004-001.pdf.

Ballesteros, G.; Cabrera, M.; Echevarrías, J. L.; Lorenzo, C. J.; Raya, C.; TORRES-EsquiVIAS, J. A.; VIEDMA, C. (2008). Tarro canelo, cerceta pardilla, porrón pardo, malvasía cabeciblanca y focha moruna en España. Población en 2007 y método de censo. Madrid, SEO/BirdLife.

BERNIS, F. (1972): «Breve reseña geográfica, migratológica y demográfica sobre algunas aves acuáticas censadas». Ardeola, n 17-18: 207-230.

BIRDLIFE INTERNATIONAL (2004) Birds in Europe: population, estimates, trends and conservation status. Cambridge (UK), BirdLife International.

CANO, L.S.; HernándeZ, J.M. (2003). «Cigüeña negra Ciconia nigra» en Atlas de las Aves Reproductoras de España (Martí, R. y Del Moral, J.C., eds.). Madrid, Dirección General de Conservación de la Naturaleza-SEO/BirdLife, 120-121.

CANo, L.S.; HeRnándEZ, J.M. (2004). «Cigüeña negra Ciconia nigra» en Libro Rojo de las Aves de España (MAdroño, A.; GonZÁlez, C.; Atienza, J.C., eds.). Madrid, Dirección General para la Biodiversidad-SEO/BirdLife, 71-73.

Casado, S.; Montes, C. (1995). Guía de los Lagos y Humedales de España Madrid, J. M. Reyero Editor.

CHG (2011). Red de control biológico. Campañas 2005-2006, 2007-2008, 2009. Ciudad Real/Badajoz, Confederación Hidrográfica del Guadiana (MARM). En línea:

http://planhidrologico2009.chguadiana.es/?chguadiana=177df140484979d38ce1 e28e44da0f46\&url=la+cuenca+hidrogr\%E1fica+redes+de+control+redes+de+co ntrol+de+calidad+red+de+control+biol\%F3gico+datos+anal\%EDticos\&corp=ch guadiana\&lang=es\&mode=view, consultado el 15/03/2011.

Cirujano, S.; Medina, L. (2002) Plantas acuáticas de las lagunas y humedales de Castilla-La Mancha. Madrid, Real Jardín Botánico (CSIC)-JCCM.

CMAJA. (2002). Plan Andaluz de Humedales. Sevilla, Consejería de Medio Ambiente (Junta de Andalucía). En línea: www.juntadeandalucia.es/ medioambiente/site/web/menuitem

DA CRuZ, H. (1986). Guía de Humedales de la Península Ibérica y Baleares. Madrid, Federación de Amigos de la Tierra (FAT).

DANTín, J. (1932). "La población de la Mancha española en el centro de su máximo endorreísmo". Boletín de la Real Sociedad Geográfica Nacional, nº 72:25-45.

DANTín, J. (1940). "La aridez y el endorreísmo en España. El endorreísmo bético". Estudios Geográficos, no 23: 269-312.

De le Court, C.; MAÑEZ, M.; GarcíA, L.; Garrido, H.; IBÁÑEZ, F. (2004). «Espátula común Platalea leucorodia» en Libro Rojo de las Aves de España (Madroño, A.; GonZÁlez, C.; ATIEnZA, J.C., eds.). Madrid, Dirección General para la Biodiversidad-SEO/BirdLife, 76-79.

Florín, M.; Montes, C.; Rueda, F. (1993). “Origin, hydrologic functioning and morphometric characteristics of small, shallow, semiarid lakes (lagunas) in la Mancha, Central Spain". Wetlands, nº 13 (4):247-259. 
Florín, M. (2001). "Ecología y Conservación de los Humedales Manchegos" en La Mancha Húmeda. Toledo, Instituto de Investigaciones y Estudios Toledanos (Diputación Provincial de Toledo), 65-92.

Gaston, K.J. (1996). «What is biodiversity?» en Biodiversity: a biology of numbers and difference (GASTON, K.J., ed.). Oxford (U.K.), Blackwell Science Ltd., 1-9.

Green, A. J.; El Hamzaoui, M.; El Agbani, M. A.; Franchimont, J. (2002). «The conservation status of Moroccan wetlands with particular reference to waterbirds and to changes since 1978». Biological Conservation, $\mathrm{n}^{\circ}$ 104: 71-82

GreEN, A. J.; ECHEVARRÍAS, J. L.; FERRÁNDEZ, M. (2003). «Cerceta pardilla Marmaronetta angustirostris» en Atlas de las Aves Reproductoras de España (MARTÍ, R. y Del Moral, J.C., eds.). Madrid, Dirección General de Conservación de la Naturaleza-SEO/BirdLife, 144-145.

GreEN, A. J.; ECHEVARRÍAS, J. L.; FERRÁNDEZ, M. (2004). «Cerceta pardilla Marmaronetta angustirostris» en Libro Rojo de las Aves de España (MADROÑO, A.; GonzÁlez, C.; ATIENZA, J.C., eds.). Madrid, Dirección General para la Biodiversidad-SEO/BirdLife, 100-104.

Gullick, T. M. y JiMÉNEZ, J. (1991). «Zarapito real Numenius arquata». Noticiario Ornitológico, Ardeola, 38: 331.

Higueras ARnal, A. (2003). Teoría y método de la Geografía. Introducción al Análisis Geográfico Regional. Zaragoza, Prensas Universitarias de Zaragoza.

HorTAs, F.; DíAZ, J.A.; BerTOLERO, A. (2003). «Archibebe común Tringa totanus» en Atlas de las Aves Reproductoras de España (MARTí, R. y DEL MORAL, J.C., eds.). Madrid, Dirección General de Conservación de la NaturalezaSEO/BirdLife, 260-261.

HoRTAs, F. (2004). «Aguja colinegra Limosa limosa» en Libro Rojo de las Aves de España (MADroño, A.; GonZÁleZ, C.; ATIENZA, J.C., eds.). Madrid, Dirección General para la Biodiversidad-SEO/BirdLife, 234-235.

HORTAS, F.; DÍAZ, J.A.; BERTOLERO, A. (2004). «Archibebe común Tringa totanus» en Libro Rojo de las Aves de España (MADROÑo, A.; GonZÁleZ, C.; ATIENZA, J.C., eds.). Madrid, Dirección General para la Biodiversidad-SEO/BirdLife, 237239.

JCCM. (2009). Propuesta de modificación de la Reserva de la Biosfera de La Mancha Húmeda. Toledo, Red de Áreas Protegidas de Castilla-La Mancha (JCCM)MAB (UNESCO).

JCCM (2011). Servicio Integral de Asesoramiento al Regante. Albacete, Consejería de Agricultura y Medio Ambiente (JCCM)-CREA (UCLM). En línea: http://crea. uclm.es/siar/datmeteo/datos hist.php (consultado el 01/02/2011)

JiMÉNEZ, J.; DEl MoRAL, A.; MORILlO, C.; SÁNCHEZ, M. J. 1992. Las aves del Parque Nacional de las Tablas de Daimiel y otros humedales manchegos. Barcelona, Lynx Edicions.

LÓPEZ DE CARRIón, M.; MENOR, A. (2003): «La Cigüeña Blanca en la provincia de Toledo. Censo de 1998 y evolución poblacional» en Actas del Congreso sobre la Naturaleza en la Provincia de Toledo. Toledo, Instituto Provincial de Investigaciones y Estudios Toledanos (Diputación Provincial), 235-243.

Madroño, A.; PAlacios, C. J.; DE JuANA, E., (1992). «La migración de la Cigüeña negra Ciconia nigra a través de España peninsular». Ardeola, no 39 (1): 9-13. 
Madroño, A.; GonZÁlez, C.; AtienZA, J.C., eds. (2005). Libro Rojo de las Aves de España. Madrid, Dirección General para la Biodiversidad-SEO/BirdLife.

MÁÑEZ, M.; RENDÓN-MARTOS, M., eds. (2009). El morito, la espátula y el flamenco en España. Población en 2007 y método de censo. Madrid, SEO/BirdLife.

Margalef, R. (1947). Limnosociología. Madrid, Monografías de Ciencias Modernas.

MARGALEF, R. (1987). «Teoría y modelado de los ecosistemas fluctuantes» en Seminario sobre Bases Científicas para la protección de los humedales en España. (Llamas, R., ed.). Madrid, Real Academia de Ciencias Exactas, Físicas y Naturales, 31-42.

Martí, R.; Del Moral, J.C. (2003). Atlas de las aves reproductoras de España. Madrid, Dirección General de Conservación de la Naturaleza-Sociedad Española de Ornitología.

Molina, B.; Del Moral, J. C. (2005). La cigüeña blanca en España. VI Censo Internacional (2004). Madrid, SEO/BirdLife.

NoguÉS-BRAVo, D. (2003). «La distribución especial de la biodiversidad. Conceptos y métodos». Cuadernos de Investigación Geográfica, nº 29: 67-82.

PAlomino, D.; MolinA, B., eds. (2009). Aves acuáticas reproductoras en España. Población en 2007 y método de censo. Madrid, SEO/BirdLife.

Peinado, M. (2000). «Humedales de la Mancha. Clima» En Humedales de Ciudad Real (GARCÍA CANSECO, V., coord.). Salamaca, Esfagnos S.L.

PÉREZ-ARANDA, D.; MATUTE, J. (2003). «Avefría Vanellus vanellus» en Atlas de las Aves Reproductoras de España (MARTí, R. y DEL MORAL, J.C., eds.). Madrid, Dirección General de Conservación de la Naturaleza-SEO/BirdLife, 380-381.

Plans, P. 1969. «Problemas del endorreísmo español». Revista de la Real Academia de Ciencias Exactas, Físicas y Naturales de Madrid, nº 63:271-309.

PRIETA, J.; Del MoRAL, J. C. (2008). La grulla común invernante en España. Población en 2007 y método de censo. Madrid, SEO/BirdLife.

Rubio ReCiO, J.M.; BEJARANO, R. (2001). «La cenicienta Zoogeografía: ¿qué hacer?» en Espacio natural y dinámicas territoriales. Homenaje al Dr. D. Jesús García Fernández (Manero, F., coord.). Valladolid, Universidad de Valladolid, 295-302.

SÁnchez, G.; Chicote, A.; Gosálvez, R.U.; Gil-Delgado, J.A.; Camps, C.; CAMACHO, A.; Florín, M. 2008. «Cambios ecológicos en una laguna salina de interior causados por una supercélula meteorológica», Comunicación en XIV Congreso de la Asociación Ibérica de Limnología. Programa Final. Libro de Resúmenes (Huelva, 9 al 12 de septiembre de 2008). Huelva, Universidad de Huelva-Asociación Ibérica de Limnología, 151.

SEO/BIRDLIFE. (2002). «Libro rojo de las aves de España» en Atlas de los vertebrados españoles: Aves reproductoras (SEO/Birdlife). Madrid, DGCONA (MMA).

TELLERÍA, J.L. (1986). Manual para el censo de los Vertebrados Terrestres. Madrid, Ed. Raíces.

THORUP, O. (2006). Breeding waders in Europe 2000. International Wader Studies 14. Thetford (UK), International Wader Study Group.

TORRES-ESQUIVIAS, J. A. (2004). «Malvasía cabeciblanca Oxyura melanocephala» en Atlas de las Aves Reproductoras de España (MARTí, R. y DeL MORAL, J.C., 
eds.). Madrid, Dirección General de Conservación de la NaturalezaSEO/BirdLife, 199-202.

TUCKER, G.M.; HEATH, M.F. (1994). Birds in Europe: their conservation status. Birdlife Conservation Series $n^{\circ}$ 3. Cambridge (U.K.), Birdlife International

Velasco, T. (2003) «La invernada de aves acuáticas en los humedales de la provincia de Cuenca. Resultados del período 1993-2003». Cuadernos de investigación $\mathrm{n}^{\circ} 2$. Madrid, Náyade editorial.

Velasco, T. (2006). Las aves acuáticas de los Humedales de Alcázar de San Juan (Ciudad Real). Alcázar de San Juan ( Ciudad Real), Ayuntamiento de Alcázar de San Juan y Aguas de Alcázar, S.A.

Velasco, T.; Molina, P. (2003). «Aves acuáticas nidificantes en los humedales de la provincia de Cuenca. Resultados del período 1993-2003». Cuadernos de investigación $\mathrm{n}^{\circ} 3$. Madrid, Náyade editorial.

Velasco, T.; Torralvo, C.; Carrasco, M.; Pérez, J. L.; Abarca, L. J.; Ruiz, J. M. (2007). «Primeros datos sobre reproducción de espátula (Platalea leucorodia) en Castilla-La Mancha, España» en Anuario Ornitológico de Ciudad Real 20042005 (CASAs, F.; Arredondo, A. y J. LóPEZ-JAMAR, eds.). Ciudad Real, SEOCiudad Real, 211-218.

Vicente, E.; Miracle, RM.; Armengol, J.; CAmacho, A.; Roca, J.; Soria, JM.; SEndra, M.D.; SANChos, D.; Rojo, C.; Boronat, M.D.; OrTEGa, E.; Rico, E.; GarcíA-AvilÉs, J.; RUEDA, J. (1998). Estudio limnológico de 28 humedales de Castilla-La Mancha como base para la elaboración del Plan de Ordenación de los Recursos Naturales. Toledo, JCCM-Unidad de Investigación de Limnología (Unversitat de València). 